2014s-42

\title{
Dispatching after Producing: The Supply of Non-Renewable Resources
}

\author{
Julien Daubanes, Pierre Lasserre
}

\begin{tabular}{c}
\hline Série Scientifique \\
Scientific Series
\end{tabular}

\section{Montréal}

Octobre 2014

(C) 2014 Julien Daubanes, Pierre Lasserre. Tous droits réservés. All rights reserved. Reproduction partielle permise avec citation du document source, incluant la notice (C).

Short sections may be quoted without explicit permission, if full credit, including (C) notice, is given to the source.
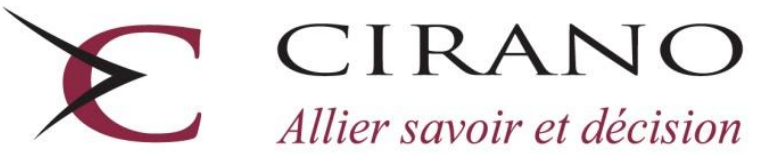

Allier savoir et décision

Centre interuniversitaire de recherche en analyse des organisations 


\section{CIRANO}

Le CIRANO est un organisme sans but lucratif constitué en vertu de la Loi des compagnies du Québec. Le financement de son infrastructure et de ses activités de recherche provient des cotisations de ses organisations-membres, d'une subvention d'infrastructure du Ministère de l'Économie, de l'Innovation et des Exportations, de même que des subventions et mandats obtenus par ses équipes de recherche.

CIRANO is a private non-profit organization incorporated under the Québec Companies Act. Its infrastructure and research activities are funded through fees paid by member organizations, an infrastructure grant from the Ministère de l' l'Économie, de l'Innovation et des Exportations, and grants and research mandates obtained by its research teams.

\section{Les partenaires du CIRANO}

Partenaire majeur

Ministère de l'Économie, de l'Innovation et des Exportations

\section{Partenaires corporatifs}

Autorité des marchés financiers

Banque de développement du Canada

Banque du Canada

Banque Laurentienne du Canada

Banque Nationale du Canada

Bell Canada

BMO Groupe financier

Caisse de dépôt et placement du Québec

Fédération des caisses Desjardins du Québec

Financière Sun Life, Québec

Gaz Métro

Hydro-Québec

Industrie Canada

Intact

Investissements PSP

Ministère des Finances et de l'Économie

Power Corporation du Canada

Rio Tinto Alcan

Ville de Montréal

\section{Partenaires universitaires}

École Polytechnique de Montréal

École de technologie supérieure (ÉTS)

HEC Montréal

Institut national de la recherche scientifique (INRS)

McGill University

Université Concordia

Université de Montréal

Université de Sherbrooke

Université du Québec

Université du Québec à Montréal

Université Laval

Le CIRANO collabore avec de nombreux centres et chaires de recherche universitaires dont on peut consulter la liste sur son site web.

Les cahiers de la série scientifique (CS) visent à rendre accessibles des résultats de recherche effectuée au CIRANO afin de susciter échanges et commentaires. Ces cahiers sont écrits dans le style des publications scientifiques. Les idées et les opinions émises sont sous l'unique responsabilité des auteurs et ne représentent pas nécessairement les positions du CIRANO ou de ses partenaires.

This paper presents research carried out at CIRANO and aims at encouraging discussion and comment. The observations and viewpoints expressed are the sole responsibility of the authors. They do not necessarily represent positions of CIRANO or its partners.

ISSN 2292-0838 (en ligne) 


\title{
Dispatching after Producing: The Supply of Non-Renewable Resources"
}

\author{
Julien Daubanes ${ }^{\dagger}$, Pierre Lasserre
}

\begin{abstract}
Résumé/abstract
There exists no formal treatment of non-renewable resource (NRR) supply, systematically deriving quantity as function of price. We establish instantaneous restricted (fixed reserves) and unrestricted NRR supply functions. The supply of a NRR at any date and location not only depends on the local contemporary price of the resource but also on prices at all other dates and locations. Besides the usual law of supply, which characterizes the own-price effect, cross-price effects have their own law. They can be decomposed into a substitution effect and a stock compensation effect. We show that the substitution effect always dominates: a price increase at some point in space and time causes NRR supply to decrease at all other points. This new but orthodox supply setting extends to NRRs the partial equilibrium analysis of demand and supply policies. The properties of restricted and unrestricted supply functions are characterized for Hotelling (homogenous) as well as Ricardian (non homogenous) reserves, for a single deposit as well as for several deposits that endogenously come into production or cease to be active.
\end{abstract}

Mots clés/Keywords: Allocating inventories, allocating reserves, supply theory, price effect, substitution effect, stock compensation effect; green paradox; spatial leakage

Codes JEL : Q38; D21: H22

\footnotetext{
* An earlier draft of this paper has been presented in various seminars and conferences: Montreal Natural Resources and Environmental Economics Workshop; SURED 2012; EAERE 2012 in Prague; CESifo Venice Workshop 2012; CREE 2012 at University of British Columbia; Toulouse School of Economics; Université du Québec à Montréal; Université de Savoie; Pre-conference of EAERE 2013 in Toulouse in Honor of Michel Moreaux; Paris School of Economics; Geneva Graduate Institute. Particular thanks go to Gérard Gaudet, André Grimaud, Michael Hoel, Sean Horan, Matti Liski, Ngo Van Long, Jérémy Laurent-Lucchetti, David Martimort, Charles Mason, Rick van der Ploeg, Debraj Ray, François Salanié, Steve Salant, Hans-Werner Sinn and Cees Withagen. Financial support from the Social Science and Humanities Research Council of Canada, the Fonds Québécois de recherche pour les sciences et la culture, the CIREQ and the CESifo is gratefully acknowledged. ${ }^{\dagger}$ Center of Economic Research at ETH Zurich. E-mail address: jdaubanes@ethz.ch

* Département des sciences économiques at UQAM, CIRANO and CIREQ. E-mail address: lasserre.pierre@uqam.ca
} 


\section{Introduction}

A basic tenet of the theory of supply is the exogeneity of price. We study the supply of commodities that need to be produced before being allocated to various uses in space and time. The path of prices over time and their distribution in space are taken as parametric and we study the properties of supply functions, that is the effect on the quantity supplied at any date or location of a price change occurring at any date or location.

While such supply functions that depend on many prices arise in numerous situations, we focus on non-renewable resources (hereafter NRRs) both for concreteness and because the need of a systematic treatment of supply is blatant in that case. Nonetheless our results apply, or can easily be adapted, to many other commodities. Surprisingly, such a highly orthodox approach is new. The literature treats prices as constant or as a single-parameter path, or alternatively studies the intersection of supply and demand without any prior treatment of supply. By establishing comparative static properties, our treatment extends to NRRs the time-honored partial equilibrium method of shifting demand and supply curves. It has immediate applications to the green paradox, spatial carbon leakages or NRR extraction responses to various policies.

The question of NRR supply has a long history and remains very contemporary. It was first addressed by Gray (1914) and formally undertaken by Burness (1976). They specifically inquired about the effect on the extraction path of changing the exogenous price, assumed to be constant throughout the extraction period. Sweeney (1993) later attempted to reconcile NRR supply with conventional supply theory, by deriving resource supply as a function of the contemporary producer price. He stated that "static supply functions, so typical in most economic analysis, are inconsistent with optimal extraction of depletable resources." (p. 780) but left the task unfinished.

The recent interest in the green paradox and carbon leakage has given rise to a number of contributions that focus in various ways on the effects across time and/or space of policy decisions implemented at various future dates and locations. The first ones (Long and Sinn, 1985; Sinn, 2008) assumed reserves to be homogenous and given rather than heterogenous and produced (by exploration and development). More recent contributions 
relax these assumptions in various ways. Hoel (2012), van der Ploeg and Withagen (2012a, 2012b, 2014) and Grafton, Kompas and Long (2012) consider that some part of the resource may be left unexploited after a demand-restricting policy change, due to heterogeneity in extraction costs. The same is true in the paper by Fischer and Salant (2013) who also consider carbon leakage and pay particular attention to the type of policy used to reduce NRR demand.

Harstad (2012) focuses on supply policy. He shows that buying up and sterilizing NRR reserves will reduce consumption and emissions, whether supply is static as in the core of the paper, or dynamic as in the two-period extension (p. 97). Venables (2014) offers a dynamic theoretical treatment of NRR supply. In the long run, supply is determined by reserves developed within an optimal investment process. However, the equilibrium market price is assumed to grow at some constant rate. Golosov, Hassler, Krusell, and Tsyvinski (2014) consider a supply taxation policy in equilibrium. Their formulation of the NRR supply side comes closest to the one offered in this paper. In the decentralized equilibrium, NRR producers maximize profits given prices under a constraint imposed by finite reserves. However they do not derive supply functions nor do they consider such long-run features as the endogeneity of reserves or the endogeneity of deposit development dates as we do in this paper.

In the textbook formulation of a supply function, producers take the price as given and choose production to maximize profits. In this paper, supply at any given date and location depends on exogenous prices over time and at various locations. We distinguish between short-run and long-run supply functions according to whether reserves (the total production to be dispatched) are given or endogenous (McFadden, 1978), and we study the fundamental properties of these functions.

When total cumulative production is given, an exogenous price change occurring at any date modifies the marginal profit from extracting at that date relative to other dates and thus entails a pure intertemporal substitution effect; this is the mechanism of the green paradox as initially formulated by Long and Sinn. A change in the resource price path faced by producers may also affect ultimately exploited reserves. We call this the 
stock-compensation effect and we show in Section 2 that the substitution effect dominates the compensation effect under standard assumptions. Consider an increase in price at some particular date leaving prices at all other dates unchanged; the pure intertemporal substitution effect increases supply at that date and reduces supply at all other dates; the stock effect results in an increase in ultimately extracted reserves. It follows that supply at the date of the price rise increases as the stock effect and the substitution effect work in the same direction; this is the NRR version of the law of supply. At all other dates, since the substitution effect dominates, it follows that supply diminishes despite the stock effect; this may be called the law of intertemporal substitution in NRR supply.

When the time dimension is combined with a space dimension as in Fischer and Salant (2013), the same result applies to a price change occurring at a point in space and time. Thus the intertemporal substitution effect is accompanied by an analogous spatial substitution effect: a price rise at any point in space and time reduces supply at all other points.

The dependence on a vector of prices, as well as the substitution and compensation effects are reminiscent of demand theory: NRR producers allocate a stock of resource to different dates and outlets in a way that is comparable to the way consumers allocate their income to different expenditures on different goods. The time space and spatial space play a similar role as the good space in static demand. The stock of reserves is not unlike the budget constraint in demand theory, as both limit what can be allocated to alternative supplies or to expenditures on alternative goods; furthermore, these constraints are both affected by prices, although by different channels. ${ }^{1}$ The law of supply always applies: unlike the Giffen paradox, the supply of a NRR always increases if its price rises. Similarly, inferior goods have no counterpart in NRR supply: given a price vector, supply does not diminish at any date if reserves are exogenously increased.

The production to be dispatched or the reserves to be extracted are costly and must be constituted prior to dispatching or extraction. We assume that the stock of reserves is produced via exploration and development efforts that are sensitive to the rent accruing to

\footnotetext{
${ }^{1}$ As is well known, the analogy between supply and demand is not an isomorphism; for one thing preferences are price independent unlike profits.
} 
the extractor during the exploitation phase. This is how prices at any date and location determine initial reserves in the long run and cause both the stock compensation and the substitution effect mentioned earlier. Furthermore, the constitution of reserves is necessarily subject to decreasing returns to scale as exploration prospects are finite. If such were not the case, NRRs would be indefinitely reproducible like some conventional commodities are in the long run. The resource rent would reduce to the constant quasirent associated with expenditures in exploration and reserve development, which would be insensitive to NRR prices. In that case, long-run supply at any date and location only depends on prices at that date; there are no intertemporal cross-price effects and supply is intertemporally separable.

It is customary to use the apparatus of supply and demand to study policies. This is the realm of partial equilibrium analysis. Applying this apparatus to NRR markets requires taking into account the intertemporal nature of NRR supply and its properties. We illustrate the procedure in Section 3 by first presenting three examples: resource taxation; a reserve-sterilization policy; a demand-reduction policy. Then we generalize the procedure to generic demand and supply policies, before considering policies with a spatial dimension and with market power.

NRR extraction technologies are often peculiar; extraction costs may depend on past cumulative extraction and may reflect spatial or geological constraints. We give due consideration to such resource heterogeneity issues in Section 4, with details in the Appendix. The output supplied remains homogenous, but the conditions of its production may vary, especially due to variations in the quality or accessibility of the reserves. We consider the two major alternative approaches that have been used in the literature to deal with such resource heterogeneity, each corresponding to a particular cost structure. One is the model where the cost of extraction increases with cumulative extraction, under the assumption that resources of easier access or higher quality are exploited first. This model has been initiated by Hotelling (1931) and Gordon (1967), and further perfected among others by Weitzman (1976) and Salant, Eswaran and Lewis (1983). It is being used in a recent literature that investigates the green paradox (e.g. Gerlagh, 2011; Hoel, 2012; 
van der Ploeg and Withagen, 2012a, 2012b, 2014; Grafton, Kompas and Long, 2012). It is also invoked in the integrated assessment model of Golosov et al. (2014). In variants associated with the energy literature (Adelman, 1990, 1993) such as Venables (2014), extraction costs increase with the rate of depletion and are "iceberg", using up the stock of reserves itself.

The second approach to resource heterogeneity, which may be attributed to Herfindahl (1967), considers a multiplicity of different deposits; it has given rise to a series of papers that study the optimum sequence and possible overlap of deposit exploitation (e.g. Amigues, Favard, Gaudet and Moreaux, 1998; Gaudet and Lasserre, 2011; Salant, 2013). In the version analyzed here, deposits have different costs of extraction and different costs of exploration and development; the timing of deposit development and exploitation is endogenous and is part of the producer's supply problem. ${ }^{2}$

Again we show that the substitution effect dominates the stock compensation effect under both approaches, although the result takes a somewhat diluted form. In the Hotelling-Gordon model with stock effects, we show that both the stock and the substitution effect materialize in terms of cumulative supply at all dates and locations although not necessarily in terms of supply flows. In the multiple-deposit model, we consider supply at individual deposits and also aggregate supply. In the long run, a change in supply occurs when the flow of production from an existing deposit changes; but supply may also change when a deposit is left undeveloped rather than being exploited or vice versa. It is shown that a price rise at any date after the planned opening of a deposit either leaves the opening date of that deposit unchanged, or postpones it but never accelerates it. Meanwhile it causes extraction from deposits already in production to diminish before the price rise. Thus at the aggregate level, the substitution effect is reinforced and dominates the stock compensation effect until at least the first new, postponed, opening date. Over that initial period, supply is always reduced following an increase in future price.

\footnotetext{
${ }^{2}$ In the computable version of their integrated assessment model, Golosov et al. (2014) use three deposits with different costs of extraction and no (reserve) stock effect on these costs. They also assume reserves to be exogenous.
} 
We conclude by putting the results in perspective, reiterating their theoretical and policy relevance, and highlighting their applicability to all commodities that must be produced prior to being dispatched in space or time.

\section{A Synthetic Theory of Non-Renewable Resource Supply}

\section{2.a A Simple Model}

A quantity $x_{t} \geq 0$ of a NRR is supplied at each of an infinite countable set of dates $t=0,1,2, \ldots$ The initial stock $X>0$ of the resource is finite and treated as exogenous at this stage, with $\sum_{t \geq 0} x_{t} \leq X$. The present-value producer price is denoted by $p_{t} \geq 0$. The stream of prices $p \equiv\left(p_{t}\right)_{t \geq 0}$ is taken as given by the producers and treated as exogenous at this stage. ${ }^{3}$ The present-value cost of producing a quantity $x_{t}$ is denoted by $C_{t}\left(x_{t}\right)$, where the function $C_{t}$ may be time varying, is increasing and twice differentiable, and satisfies $C_{t}^{\prime \prime}\left(x_{t}\right)>0$. For simplicity, we also assume ${ }^{4} C_{t}(0)=0$ for all dates $t \geq 0$ and $C_{t}^{\prime}(0)<p_{t}$ for at least one date so that some exploitation is warranted.

The stock of reserves to be exploited does not become available without some prior exploration and development efforts. Although exploration and exploitation often take place simultaneously at the aggregate level (e.g. Pindyck, 1978, and Quyen, 1988; see Cairns, 1990, for a comprehensive survey of related contributions), at the microeconomic level of a deposit they occur in a sequence, as in Gaudet and Lasserre (1988) and Fischer and Laxminarayan (2005). This way to model the supply of reserves is particularly adapted to the problem under study because it provides a simple and natural way to isolate the effect of an anticipated price change on the size of the exploited stock at the firm level. Specifically, assume that the present-value cost $E(X)$ of developing an initial, exploitable stock $X$ at date 0 is twice differentiable, increasing, strictly convex, and satisfies $E(0)=0$ and $E^{\prime}(0)=0$. The property $E^{\prime}(0)=0$ that the marginal cost of reserve development is zero at the origin is introduced because it is sufficient to ensure that a positive amount of reserves is developed. It thus rules out uninteresting situations

\footnotetext{
${ }^{3}$ We will show how the results carry over to a partial-equilibrium setting where prices are endogenously determined on markets.

${ }^{4}$ The qualitative results follow through in presence of a fixed cost or lump sum tax.
} 
where resource prices do not warrant the production of any reserves.

Since the development of reserves is costly, the optimum plans of the producers will always bind the exhaustibility constraint. In other words, leaving part of the developed stock ultimately unexploited does not maximize cumulative net discounted revenues. For a given price sequence $p$, the cumulative value function corresponding to a producer's optimum is

$$
\max _{\left(x_{t}\right)_{t \geq 0}, X} \sum_{t \geq 0}\left(p_{t} x_{t}-C_{t}\left(x_{t}\right)\right)-E(X)
$$

subject to

$$
\sum_{t \geq 0} x_{t}=X .
$$

Denoting by $\lambda$ the Lagrange multiplier associated with constraint (2), the necessary first-order conditions characterizing the optimum extraction path are

$$
p_{t}-C_{t}^{\prime}\left(x_{t}\right) \leq \lambda \text { with }\left(p_{t}-C_{t}^{\prime}\left(x_{t}\right)-\lambda\right) x_{t}=0, \forall t \geq 0
$$

i.e. at dates where extraction is strictly positive ${ }^{5}$

$$
p_{t}-C_{t}^{\prime}\left(x_{t}\right)=\lambda, \forall t \geq 0, x_{t}>0
$$

For the choice of initial reserves, the first-order condition is

$$
E^{\prime}(X)=\lambda
$$

Expression (4) is the Hotelling rule stating that the marginal profit from extraction must be constant in present value over the period of active exploitation, equal to $\lambda$, the unit present value of reserves underground, called the Hotelling scarcity rent. (5) is a standard supply relationship that sets marginal cost equal to price. The price in this case is the unit scarcity rent and is defined implicitly; in other words reserves are the output of a production process whose technology is described by the cost function $E$. However reserves are not like conventional goods that can be produced under constant returns to

\footnotetext{
${ }^{5}$ If the price is too low at some date, production may be interrupted before exhaustion, and start again once prices are high enough. The first-order condition during production interruptions (it must also hold after exhaustion) is $p_{t}-C_{t}^{\prime}\left(x_{t}\right) \leq \lambda$ for all $x_{t} \geq 0$, that is equivalently $p_{t}-C_{t}^{\prime}(0) \leq \lambda$.
} 
scale, because of the scarcity of exploration prospects. The supply of reserves is thus a strictly increasing function of the rent: ${ }^{6}$

$$
X=X(\lambda) \equiv E^{\prime-1}(\lambda)
$$

The Hotelling rule (4) implicitly defines the solution of Problem (1)-(2) as a series of functions $x_{t}$ giving extraction at each date. ${ }^{7}$ Each function is increasing in the current price $p_{t}$ and decreasing in the rent $\lambda$ :

$$
x_{t}=x_{t}\left(p_{t}, \lambda\right), \forall t \geq 0
$$

As Sweeney (1993) noted, functions like (7) can be interpreted as conventional static supply functions, whose only arguments are the price $p_{t}$ of the extracted resource and the reserve price $\lambda$. However $\lambda$ is not a conventional price; unlike standard price parameters, it corresponds to the "shadow" or implicit valuation of reserve units and thus is endogenous to the resource producer problem. Formulating regular supply functions consequently further requires expressing the rent $\lambda$ as a function of the vector of exogenous prices $p$.

\section{2.b Short-Run (Restricted) NRR Supply Functions}

Treating the stock of initial reserves as given at this stage and combining all relations (7) into (2), we obtain that the rent is a function increasing in all prices in $p \equiv\left(p_{t}\right)_{t \geq 0}$ and decreasing in the stock $X$; we will denote that function with a tilde, and will do so for all functions of given reserves:

$$
\lambda=\widetilde{\lambda}(p, X)
$$

Substituting (8) into (7) gives the restricted supply functions (McFadden, 1978), one at each date: ${ }^{8}$

$$
x_{t}=\widetilde{x}_{t}(p, X) \equiv x_{t}\left(p_{t}, \widetilde{\lambda}(p, X)\right), \forall t \geq 0 .
$$

\footnotetext{
${ }^{6}$ The finiteness of exploration prospects amounts to a fixed factor being imposed on the production process. Hence reserves are produced under rising marginal costs.

${ }^{7}$ During extraction interruptions or after exhaustion, the function takes a null value.

${ }^{8} \mathrm{~A}$ standard restricted supply function depends on the output price, on the prices of variable factors, and on the quantity of at least one restricted factor. Here variable-factor prices are the prices of the factors entering the extraction technology, omitted from the notation for simplicity, and the restricted factor is the initial stock of reserves.
} 
These functions do not make use of the first-order condition for initial reserves. Conditional on the initial reserve stock $X$ and given the sequence $p$ of prices, they determine how the suppliers allocate extraction from the stock to different dates. Unlike the restricted supply of a conventional good which only depends on its own price and on the quantity of some factor, the restricted NRR supply function at $t$ further depends on resource prices at all other dates. This is so despite the fact that the same standard technological assumptions hold in the NRR and the conventional good cases: the extraction cost at one date does not depend here on the extraction cost at another date, just as the cost of producing a conventional good is independent of the cost of producing another good.

Hotelling's lemma is obtained from the optimized value function by use of the envelope theorem for constrained problems. That is, substituting (9) and (8) into the Lagrangian function associated with Problem (1)-(2) and differentiating with respect to $p_{t}$, while holding the restricted level of $X$ and its multiplier as well as all extraction rates constant, gives the restricted supply at $t .^{9}$

The restricted NRR supply function $\widetilde{x}_{t}$ for any date $t \geq 0$ is strictly increasing in $X$. Holding the reserve level unchanged, consider the partial effects of prices, that is the direct price effects. $\widetilde{x}_{t}(p, X)$ is strictly increasing in $p_{t}$ and strictly decreasing in any $p_{T}$, $T \neq t$. This can be shown as follows. By (9), $\frac{\partial \widetilde{x}_{t}(p, X)}{\partial p_{T}}=\frac{\partial x_{t}\left(p_{t}, \widetilde{\lambda}(p, X)\right)}{\partial p_{T}}+\frac{\partial x_{t}\left(p_{t}, \lambda\right)}{\partial \lambda} \frac{\partial \widetilde{\lambda}(p, X)}{\partial p_{T}}$, where the first term on the right is zero unless $T=t$, as $x_{t}\left(p_{t}, \lambda\right)$ is not directly dependent on prices other than the contemporary price. The second term is clearly negative whether $T=t$ or $T \neq t$ since $x_{t}$ decreases in $\lambda$ while $\frac{\partial \widetilde{\lambda}(p, X)}{\partial p_{T}}$ is clearly positive since a rise in the resource price at any date cannot reduce the rent. It follows that $\frac{\partial \widetilde{x}_{t}(p, X)}{\partial p_{T}}$ is negative for $T \neq t$ while a contemporary rise in price involves two effects working in opposite directions. However, if extraction diminishes at all dates $t \neq T$, it must increase at $t=T$ for otherwise reserves would not be exhausted, which would be suboptimal as already discussed: the law of supply also applies to restricted supply. Consequently, in case of a

\footnotetext{
${ }^{9}$ Hotelling's lemma is obtained similarly in the case of non-restricted supply functions defined further below. The non-restricted value function is obtained by replacing the restricted level of $X$ and the rent $\lambda$ by their optimized values $X^{*}(p)$ and $\lambda^{*}(p)$ defined shortly below.
} 
contemporary price rise, the direct price effect given by the first term must dominate the second term that operates via the resource rent.

\section{2.c Long-Run (Unrestricted) NRR Supply Functions}

Consider the choice of initial reserves. While (6) is a standard stock supply relation, the price $\lambda$ is not a standard exogenous price but an endogenous variable. The supply of reserves at the producer's optimum in Problem (1)-(2) can be expressed as a function of exogenous prices. The value of the unit rent at the producer's optimum satisfies $\lambda=\widetilde{\lambda}(p, X)$. By (6), the optimum amount of reserves satisfies $X=X(\lambda)=X(\widetilde{\lambda}(p, X))$, which implicitly defines $X$ and $\lambda$ as functions of $p$ :

$$
X=X^{*}(p) \text { and } \lambda=\lambda^{*}(p) \equiv \tilde{\lambda}\left(p, X^{*}(p)\right)
$$

Thus the supply of reserves depends on the whole sequence of resource prices, although this can be summarized into one single rent. ${ }^{10}$

Restricted supply or factor demand as well as restricted cost or profit functions are usually interpreted as representations of the short run. In the long run, the restricted factor is variable. This interpretation is adequate here, exploration and reserve development being analogous to capital investment. Just as capital goods are produced, reserves in (10) are the outcome of a production process. Then they are used as a factor of production in the resource production process that generates the restricted supply (9). ${ }^{11}$

The optimal (unrestricted) NRR supply functions are defined as

$$
x_{t}^{*}(p) \equiv \widetilde{x}_{t}\left(p, X^{*}(p)\right), \forall t \geq 0 .
$$

Like the restricted supply, the (unrestricted) supply of a NRR differs from a conventional

\footnotetext{
${ }^{10}$ As before, factor prices are omitted for notational simplicity from the reserve-supply function. They are the prices of the factors entering the extraction process because they affect the optimum rent, but also the prices of the factors entering the exploration and development process which are omitted arguments of the $E$ cost function.

${ }^{11}$ Although this is not usually modeled, capital does get depleted (worn out) by production at a rate that depends on the rate of production. However conventional capital can be replenished in a plant while this is not, or only partially, true of the reserves of a mine. On the related subjects of resource substitution and sustainability, see the huge literature initiated with the 1974 Symposium of the Review of Economic Studies.
} 
supply function under identical standard technological assumptions in that it not only depends on its own price, the current price, but also on the prices at all other dates.

The comparative static properties of NRR supply are thus defined over a wider set of variables than those of a conventional supply function. With conventional supply functions, attention is usually limited to the law of supply, the effect of a change in the price of the good supplied. ${ }^{12}$ With NRR, supply cross-price elasticities, the effect on supply at $t$ of changes in prices at other dates, are also of theoretical interest: as we shall see, they obey their own law.

The resource literature has seldom considered such exogenous price changes, and never in a systematic treatment of supply. One exception is Burness (1976) who forced prices to be constant in current value and investigated the effect on production of a simultaneous change in all prices. Another exercise frequent in the resource taxation literature (e.g. Dasgupta, Heal and Stiglitz, 1981) has been to ask what time profile of taxes would be neutral. Both Burness' and Dasgupta, Heal and Stiglitz's findings result from the following property of the restricted NRR supply function. Let $A \equiv(a)_{t \geq 0}$ be a vector of equal constants $a$. Assume that $a>-\widetilde{\lambda}(p, X)$ for some given level $X$. Then it is easily shown that $\widetilde{\lambda}(p+A, X)=\widetilde{\lambda}(p, X)+a$ and $\widetilde{x}_{t}(p+A, X)=\widetilde{x}_{t}(p, X)$; if $a$ is a unit tax such as a unit severance tax, this shows that the tax is neutral if it is constant in present value. This result does not extend to the unrestricted supply function, when initial reserves are allowed to adjust (Gaudet and Lasserre, 2013). Indeed, if $a<0$ so that $\widetilde{\lambda}(p+A, X)<\widetilde{\lambda}(p, X)$, the initial reserve level is lower at prices $p+a$ by (6) so that the NRR supply at all dates is reduced.

Let us now turn to standard comparative supply analysis: what is the effect of a change in price at date $T$ on supply at date $t$. One must distinguish between a change at the same date $T=t$ and a change at $T \neq t$. From (11), this decomposes into a direct price effect and a stock compensation effect:

$$
\frac{\partial x_{t}^{*}(p)}{\partial p_{T}}=\frac{\partial \widetilde{x}_{t}\left(p, X^{*}(p)\right)}{\partial p_{T}}+\frac{\partial \widetilde{x}_{t}\left(p, X^{*}(p)\right)}{\partial X} \frac{\partial X^{*}(p)}{\partial p_{T}} .
$$

When $T=t$, the total price effect may be called the own price effect; since $\widetilde{x}_{t}$ is increasing

\footnotetext{
${ }^{12}$ We ignore factor prices for simplicity.
} 
in both $p_{t}$ and $X$, and as resource prices always affect developed reserves positively, the own price effect is positive. Expression (12) when $T=t$ indicates that the law of supply holds and illustrates the Le Châtelier principle, which says that the long-run (unrestricted) elasticity is higher than the short-run (restricted) elasticity.

When $T \neq t$, the direct price effect in (12) may be called the pure substitution effect as it reflects the reallocation of an unchanged reserve stock to extraction at a date different from $T$; (9) makes clear that this substitution effect only arises via the effect of the rent on the $\widetilde{x}_{t}$ function: $\frac{\partial \widetilde{x}_{t}(p, X)}{\partial p_{T}}=\frac{\partial x_{t}\left(p_{t}, \lambda\right)}{\partial \lambda} \frac{\partial \widetilde{\lambda}(p, X)}{\partial p_{T}}$. Also by (9), the stock compensation effect works in the opposite direction and can be itself decomposed into $\frac{\partial \widetilde{x}_{t}(p, X)}{\partial X} \frac{\partial X^{*}(p)}{\partial p_{T}}=$ $\frac{\partial x_{t}(p t, \lambda)}{\partial \lambda} \frac{\partial \widetilde{\lambda}(p, X)}{\partial X} \frac{\partial X^{*}(p)}{\partial p_{T}}$ so that the total cross-price effect can be factorized as follows:

$$
\frac{\partial x_{t}^{*}(p)}{\partial p_{T}}=\frac{\partial x_{t}\left(p_{t}, \lambda^{*}(p)\right)}{\partial \lambda}\left[\frac{\partial \widetilde{\lambda}\left(p, X^{*}(p)\right)}{\partial p_{T}}+\frac{\partial \widetilde{\lambda}\left(p, X^{*}(p)\right)}{\partial X} \frac{\partial X^{*}(p)}{\partial p_{T}}\right], T \neq t,
$$

where the term between brackets is in fact the total derivative of $\widetilde{\lambda}(p, X)$ with respect to $p_{T}$, decomposed into a direct price effect at constant initial reserves, and the effect on the rent of the change in initial reserves induced by the price change. Resource prices at all dates affect the rent positively, i.e. $\frac{\partial \lambda^{*}(p)}{\partial p_{T}} \geq 0, \forall T \cdot{ }^{13}$ Consequently,

$$
\frac{\partial x_{t}^{*}(p)}{\partial p_{T}}=\frac{\partial x_{t}\left(p, \lambda^{*}(p)\right)}{\partial \lambda} \frac{\partial \lambda^{*}(p)}{\partial p_{T}} \leq 0, \forall t \neq T,
$$

implying that the stock compensation effect is never high enough to offset the pure substitution effect. ${ }^{14}$

\footnotetext{
${ }^{13}$ Formally, the definition of $X^{*}(p)=X\left(\widetilde{\lambda}\left(p, X^{*}(p)\right)\right)$ yields $\frac{\partial X^{*}(p)}{\partial p_{T}}=\frac{X^{\prime}(\lambda) \frac{\partial \tilde{\lambda}\left(p, X^{*}(p)\right)}{\partial p_{T}}}{1-\frac{\partial \lambda\left(p, X^{*}(p)\right)}{\partial X} X^{\prime}\left(\lambda^{*}(p)\right)}$, implying that the term between brackets in (13) can be factorized as $\frac{\partial \lambda^{*}(p)}{\partial p_{T}}=\frac{\partial \widetilde{\lambda}\left(p, X^{*}(p)\right)}{\partial p_{T}}\left(\frac{1}{1-\frac{\partial \tilde{\lambda}\left(p, X^{*}(p)\right)}{\partial X} X^{\prime}\left(\lambda^{*}(p)\right)}\right)$, which is positive since $\frac{\partial \widetilde{\lambda}(p, X)}{\partial X}$ is negative. By (10), it also follows that $\frac{\partial X^{*}(p)}{\partial p_{T}}$ is positive.

${ }^{14}$ We have assumed decreasing returns to the development of reserves - increasing marginal cost of development, i.e. strict convexity of the cost function $E$. This assumption reflects the finiteness of extraction and exploration prospects and is essential to the result. Suppose on the contrary that the development of reserves were subject to constant returns to scale: $E(X)=e X$. As before, $\lambda$ would give the present value of each reserve unit so that $\lambda=e$. The rent, thus determined by the technology, would then be insensitive to variations in prices $p$, and resource supply at $t$ would only depend on current resource price by (9). Constant returns to scale in the development of $X$ make all cross-price effects on extraction vanish, just like in the classical theory of supply under separable costs.
} 


\section{2.d Properties of Homogenous NRR Supply}

Besides the law of supply that characterizes the own-price effect, our analysis has established two properties that are specific to NRR supply functions: the stock effect, and the cross-price effect. Subsections 4.a and 4.b will show how those results carry over from the simple context of this section to settings that are considered richer and more realistic but less intuitive in the literature.

\section{Proposition 1 (Supply from a homogeneous single-outlet NRR deposit)}

1. Stock effect: An exogenous rise in exploitable reserves increases short-run (restricted) supply at all dates.

2. Cross-price effects: A price rise at any date $T$ reduces short-run (restricted) supply and long-run (unrestricted) supply at all dates $t \neq T$.

Although reminiscent of the decomposition of Marshallian demand, the decomposition of the change in NRR supply at $t$ following a price change at $T \neq t$ into a pure substitution effect and a stock compensation effect is not isomorphic to the Slutsky decomposition. The substitution effect and the stock compensation effect of a resource price change are illustrated in Figure 1 for the case of two periods, which corresponds to the two-good representation of demand theory. Assuming prices $p_{0}$ and $p_{1}$, point $O=\left(x_{0}, x_{1}\right)$ in Figure 1 depicts the producer optimum. Given a stock of reserves $X$, periods 0 and 1 extraction levels are chosen such that the producer reaches the highest possible two-period isoextraction-profit curve for prices $\left(p_{0}, p_{1}\right)$ (of level $\left.\bar{\pi}\right){ }^{15}$ The optimum allocation $\left(x_{0}, x_{1}\right)$ is thus at the point of tangency between the $\bar{\pi}$ iso-profit curve and the exhaustibility constraint, the -45 degree line which expresses the trade-off between quantities extracted in period 1 and quantities extracted in period 2 in such a way that $x_{0}+x_{1}=X$. Unlike

\footnotetext{
${ }^{15}$ In Figure 1, the iso-profit curves correspond to the two-period extraction profit, conditional on $X$ and before deduction of the sunk exploration cost $E(X): \bar{\pi}=\left(p_{0} x_{0}-C_{0}\left(x_{0}\right)\right)+\left(p_{1} x_{1}-C_{1}\left(x_{1}\right)\right)$. By (4), any optimum extraction is such that $p_{t}-C_{t}^{\prime}\left(x_{t}\right)=\lambda$. Thus in a neighborhood of any optimum, $p_{t}-C_{t}^{\prime}\left(x_{t}\right)>0$. In a neighborhood of an optimum, it follows from the convexity of $C_{t}$ that the slope $-\frac{p_{0}-C_{0}^{\prime}\left(x_{0}\right)}{p_{1}-C_{1}^{\prime}\left(x_{1}\right)}$ of any iso-profit curve at prices $\left(p_{0}, p_{1}\right)$ is negative, increasing in $x_{0}$ and decreasing in $x_{1}$. In Figure 1, we focus on the relevant convex parts of the iso-profit curves. On other parts, they need not be convex.
} 
the case of Marshallian demand, the slope of this linear constraint is not affected by changes in prices. Also, while prices do not affect iso-utility curves, they affect the slope of iso-profit curves: iso-profit curves may cross at different prices.

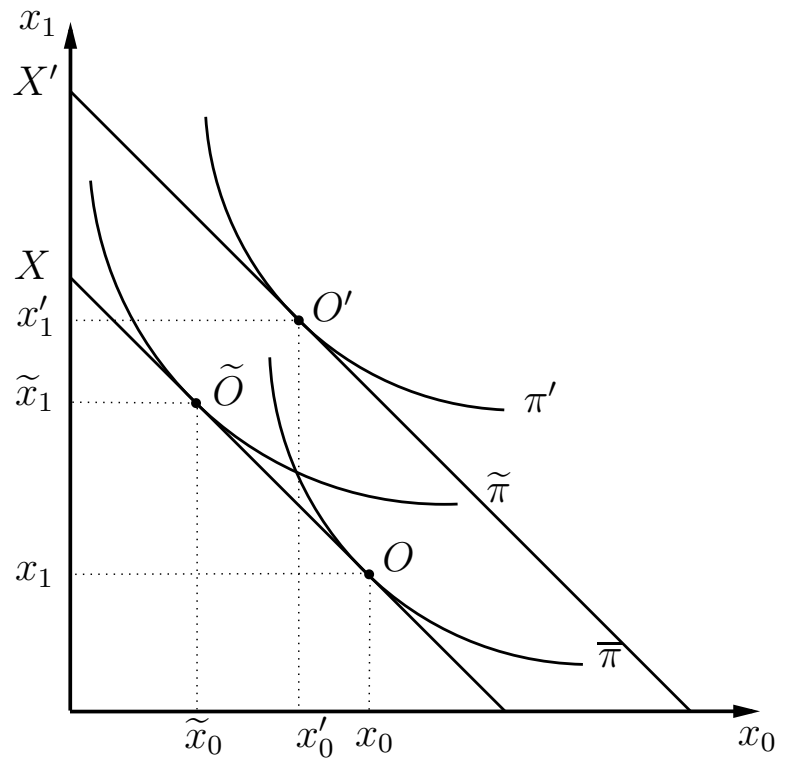

Figure 1: Price effect decomposition with $p_{1}^{\prime}>p_{1}$

Consider a rise in $p_{1}$ to $p_{1}^{\prime}>p_{1}$. The price change implies that all iso-profit curves become flatter at any given feasible level of $x_{0}$. If the stock of reserves remains unchanged at $X$, the new tangency point is along the same exhaustibility constraint and along the iso-profit curve of level $\tilde{\pi}>\bar{\pi}$, at point $\widetilde{O}$ above $O$, so that $\widetilde{x}_{0}<x_{0}$ and $\widetilde{x}_{1}>x_{1}$. The move from $O$ to $\widetilde{O}$ represents the substitution effect.

However the rise in price leads producers to increase reserve development to $X^{\prime}$. Taking this stock effect into account brings the new optimum to $O^{\prime}$. It is clear that $x_{1}^{\prime}>\widetilde{x}_{1}>x_{1}$. Unlike the Slutsky decomposition, there is no possibility of a commodity analogous to a Giffen good, whose supply would diminish as a result of a rise in its price. Moreover, in the case of NRR supply, the substitution effect always dominates the compensation effect, so that, by (14), $x_{0}^{\prime}$ must be lower than $x_{0}$ following the rise in $p_{1}$. There is no such thing as NRR supply complements; quantities extracted at different dates are always substitutes. 


\section{2.e Spatial NRR Supply}

For simplicity, we have focused so far on the dynamic interpretation of the NRR supply model. However, to the countable set of dates $t=0,1,2, \ldots$ we may add a spatial dimension indexed by $l=0,1,2, \ldots, \bar{l}$; in that formulation, the price $p_{t l}$ is the present-value producer price at date $t$ and location $l$. Location may then refer to a particular country or jurisdiction characterized by a particular price sequence, or an outlet commanding particular marketing efforts or transportation costs. The net spot revenue from selling the amount $x_{t l}$ at location $l$ at date $t$ is $p_{t l} x_{t l}-c_{t l}\left(x_{t l}\right)$, where the function $c_{t l}\left(x_{t l}\right)$ gives the cost of selling specifically in location $l$ at $t$; it may be a transportation cost, a marketing cost, etc. It is assumed that $c_{t l}$ is increasing and strictly convex. The net spot revenue from serving all locations $l=0, \ldots, \bar{l}$ at date $t$ is $\sum_{l=0, \ldots, \bar{l}}\left(p_{t l} x_{t l}-c_{t l}\left(x_{t l}\right)\right)-C_{t}\left(x_{t}\right)$, where $x_{t}=\sum_{l=0, \ldots, \bar{l}} x_{t l}$ is the quantity of NRR extracted from the deposit at date $t$ and the function $C_{t}\left(x_{t}\right)$ gives the cost of extracting and otherwise processing this quantity before dispatching it to all locations $l=0, \ldots, \bar{l}$. The cumulative value function corresponding to a producer's optimum allocation of the production, for a given matrix of prices, is

$$
\max _{\left(x_{t}\right)_{t \geq 0}\left(x_{t l}\right)_{t \geq 0, l=0, \ldots, \bar{l}}, X} \sum_{t \geq 0}\left(\sum_{l=0, \ldots, \bar{l}}\left(p_{t l} x_{t l}-c_{t l}\left(x_{t l}\right)\right)-C_{t}\left(x_{t}\right)\right)-E(X),
$$

not only subject to the exhaustibility constraint (2) as previously in Subsection 2.a, but also to the constraint

$$
\sum_{l=0, \ldots, \bar{l}} x_{t l}=x_{t}, \forall t \geq 0 .
$$

Defining $v_{t}$ as the Lagrange multiplier associated with the new constraint (16) and keeping the notation $\lambda$ for the multiplier associated with the exhaustibility constraint (2), the necessary first-order conditions characterizing the producer's optimum are

$$
p_{t l}-c_{t l}^{\prime}\left(x_{t l}\right)=v_{t}, \forall t \geq 0, \forall l=0, \ldots, \bar{l},
$$

for the allocation of the production at any date $t$ to all locations $l$, and

$$
v_{t}-C_{t}^{\prime}\left(x_{t}\right)=\lambda, \forall t \geq 0
$$


for the choice of extraction at date $t$. For simplicity, and with no consequence on the sign of the price effects to be established, we focus here on interior resource allocations where $x_{t l}>0$. The choice of initial reserves is determined by the same condition (5) as previously. Condition (18) is the counterpart of (4) in the single-location model of Subsection 2.a, except that the implicit value $v_{t}$ plays the role of the price at date $t$ in the determination of the rate of extraction. $v_{t}$ can be interpreted as the after-extraction resource rent, i.e. the implicit value of the inventory to be dispatched. Condition (17) plays the role of allocating production to outlets by equalizing the contributions to $v_{t}$ from the various locations. The analysis then unfolds as in the single-location treatment of Subsections 2.a-2.d, but in two stages rather than one, as follows.

The allocation rule (17) implicitly defines the solution as a series of functions giving the optimal quantity at each date and location

$$
x_{t l}=x_{t l}\left(p_{t l}, v_{t}\right)
$$

with the same properties as the functions defined by (7). The rule (18) similarly defines the total quantity across locations at each date $t$ as a function

$$
x_{t}=\bar{x}_{t}\left(v_{t}, \lambda\right) \text {. }
$$

The functions $\bar{x}_{t}$ have properties with respect to the implicit value $v_{t}$ and the rent $\lambda$ that are analogous to those of $(7): \bar{x}_{t}$ is increasing in the implicit price $v_{t}$ and decreasing in the rent $\lambda$.

Treating date- $t$ extraction $x_{t}$ to be dispatched across locations as given - in the same way as we took reserves $X$ to be allocated across dates as given in Subsection 2.a -, and combining all relations (19) into (16), we obtain the new multiplier $v_{t}$ as a function increasing in all components of the vector of date- $t$ prices $p_{t} \equiv\left(p_{t l}\right)_{l=0, \ldots, \bar{l}}$ and decreasing in $x_{t}$ :

$$
v_{t}=\widetilde{v}_{t}\left(p_{t}, x_{t}\right)
$$

Substituting (21) into (20) yields an implicit function identical to (7)

$$
x_{t}=x_{t}\left(p_{t}, \lambda\right)
$$


except that $p_{t}$ is a vector rather than a single price.

A rise in $p_{T L}$, i.e. a rise at any date $T \geq 0$ and location $L \in[0, \ldots, \bar{l}]$, causes the rent $\lambda$ to increase. This is true whether the size of the exploitable reserves $X$ is restricted or not. Thus, by (22), extraction decreases at all dates $t$ other than $T$. When reserves $X$ are endogenous, they increase as a result of the price rise; since all $x_{t}$ are reduced at all $t \neq T$, it follows that $x_{T}$ must increase if the sum of all $x_{t}$ is to use up reserves.

According to (19), quantities $x_{t l}$ at all locations react in the same direction to the same change in implicit value $v_{t}$. Since $x_{t}=\sum_{l=0, \ldots, \bar{l}} x_{t l}$, the reduction in $x_{t}$ at $t \neq T$ is only compatible with a rise in $v_{t}$. Thus $x_{t l}$ decreases for all $t \neq T$ and all $l$.

At date $T$ where $x_{T}$ increases as a result of the rise in $p_{T L},(20)$ makes clear that only a rise in $v_{T}$ is consistent with the increase in $\lambda$. The rise in $v_{T}$ in turn implies by (19) that $x_{T l}$ diminishes for all $l \neq L$. Since, total extraction $x_{T}$ at date $T$ rises, it must be that $x_{T L}$ rises as a result of the price rise, which illustrates the law of supply for this spatio-temporal version of the model.

\section{Proposition 2 (Supply from a homogeneous multiple-outlet NRR deposit)}

1. Stock effect: An exogenous rise in exploitable reserves increases short-run supply at all dates and locations.

2. Cross-price effects: $A$ rise in price $p_{T L}$ at any date $T$ and location $L$ reduces shortrun and long-run supply at all locations $l \neq L$ at date $T$, and at all locations $l$ at dates $t \neq T$.

In Section 3, we illustrate how the properties of NRR supply functions can be used to analyze policies in partial equilibrium. For ease of exposition, we do so using the supply functions characterized in the simple setup of this section. We will only consider more complex setups later, in Subsections 4.a and 4.b, and show that the qualitative results on supply functions and partial equilibrium analysis are almost unchanged. 


\section{$3 \quad$ Partial Equilibrium and Policy Analysis}

Having defined and characterized NRR supply functions in the standard way opens the field of all applications that rely on the demand-supply schedule, in particular the partial equilibrium analysis of economic policies. Policy-induced changes are more complex than the above analysis of supply for two main reasons. First, the policy often affects equilibrium prices indirectly, because it affects the demand for, or the supply of, the NRR; second, policy-related price changes usually take place over an extended period rather than at a single date.

Three examples are provided below: one on supply extraction taxation; one of a reserve-reduction policy; and one of a policy affecting demand and raising the issue of the green paradox. The equilibrium is determined by the intersection of supply with demand, and the supply properties established in Section 2 are used to assess the equilibrium effects of changing a policy at some date. These examples illustrate general partial-equilibrium properties of NRR markets that are gathered in Proposition 3 at the end of this section. The issue of policy duration and timing is covered in a final corollary while the section ends with a discussion of market power.

\section{3.a The Taxation of Extraction}

Suppose that prices are determined by the equilibrium of NRR demand and supply and consider a policy that penalizes supply, such as the taxation of extraction. Let net after-tax spot extraction revenues be $\Pi_{t}\left(x_{t}, p_{t}\right)=p_{t} x_{t}-C_{t}\left(x_{t}\right)-G_{t}\left(x_{t}, p_{t}\right)$, where $G_{t}\left(x_{t}, p_{t}\right)$ is the present-value tax function (Gaudet and Lasserre, 2013). When the tax is a unit or specific severance tax, $G_{t}\left(x_{t}, p_{t} ; \alpha_{t}\right)=\alpha_{t} x_{t}$; when it is an ad valorem severance tax, $G_{t}\left(x_{t}, p_{t} ; \beta_{t}\right)=\beta_{t} p_{t} x_{t}$. When policies combine those taxes, $G_{t}\left(x_{t}, p_{t} ; \alpha_{t}, \beta_{t}\right)=$ $\left(\beta_{t} p_{t}+\alpha_{t}\right) x_{t}$ and after-tax extraction revenues may be rewritten $\Pi_{t}\left(x_{t}, p_{t} ; \alpha_{t}, \beta_{t}\right)=$ $\left[p_{t}\left(1-\beta_{t}\right)-\alpha_{t}\right] x_{t}-C_{t}\left(x_{t}\right)$. Therefore, the analysis of Section 2 is only modified to the extent that the producer price in absence of supply taxation should be replaced by the after-tax producer price $p_{t}\left(1-\beta_{t}\right)-\alpha_{t}$.

Assume that date- $t$ demand $x_{t}^{D}\left(p_{t}\right)$ is strictly decreasing. For simplicity, let us focus 
on a specific $\operatorname{tax} \alpha_{T} \geq 0$ applied at some date $T \geq 0$ and only at that date: $\alpha_{t}=0$, $\forall t \neq T$. The partial-equilibrium implications of raising the tax from zero to $\alpha_{T}>0$ are depicted in Figure 2 where points $I_{T}$ and $I_{t}$ represent the initial situation at dates $T$ and $t$ respectively. Prices $p_{t}^{e}, t \geq 0$, denote pre-tax equilibrium prices. Initially assuming that prices at dates $t \neq T$ are unchanged, date- $T$ supply is reduced to $x_{T}^{*}\left(p_{T}-\alpha_{T},\left(p_{t}^{e}\right)_{t \neq T}\right)$ (Figure $2(\mathrm{a})$ ). If demand were perfectly inelastic, $p_{T}^{e}$ would increase by exactly $\alpha_{T}$ leaving the producer price $p_{T}^{e}-\alpha_{T}$ unchanged. Demand being downward sloping, $p_{T}^{e}$ varies by less than the tax change, so that the producer price decreases from $p_{T}^{e}$ to $p_{T}^{e}\left(\alpha_{T}\right)-\alpha_{T}<p_{T}^{e}$. A lower producer price at date $T$ reduces the quantity supplied at $T$ but also contributes to a positive shift in the supply curves at all other dates $t \neq T$ by Proposition 1 , and thus to a drop in price at these dates. In turn, at any date $t^{\prime} \neq T$, reduced producer prices at other dates $t$ also different from $T$ further reinforce the upward shift in the supply curve (Figure 2(b)). Fully adjusted prices $p_{t}^{e \prime}$ are thus lower and quantities supplied are higher than before the tax at all dates $t \neq T$. It follows that there is a feedback at $T$ which shifts supply up to $x_{T}^{*}\left(p_{T}-\alpha_{T},\left(p_{t}^{e^{\prime}}\right)_{t \neq T}\right)$. Yet, the latter upward shift in date- $T$ supply does not compensate the initial downward shift: indeed, when reserves are fixed as in the short-run, the increase in quantities at all other dates $t \neq T$ implies that supply must decrease at date $T$; in the long-run, exploitable reserves are lowered by the tax, so that extraction at date $T$ is further reduced.

\section{3.b Reserve-Reducing Policy}

Consider a supply policy that aims at reducing exploitable reserves $X$, for example in the spirit of Harstad (2012) by buying up and sterilizing some of these reserves. If initial exploitable reserves were fixed, resource supply would be directly given by restricted functions $\widetilde{x}_{t}(p, X)$ defined by $(9)$, that are all increasing in reserves according to Proposition 1. Thus any policy that reduces reserves from a level $X$ to $X^{\prime}=X-\Omega$ causes production to diminish at all dates. Partial-equilibrium implications are obvious, as illustrated in Figure 3 where point $I_{t}$ represents the initial situation at date $t$. At all dates $t \geq 0$, instantaneous supplies $\widetilde{x}_{t}(p, X)$ meet instantaneous downward-sloping demands $x_{t}^{D}\left(p_{t}\right)$ and determine equilibrium prices $p_{t}^{e}$. For a change in exploitable reserves from $X$ down to $X^{\prime}$ 


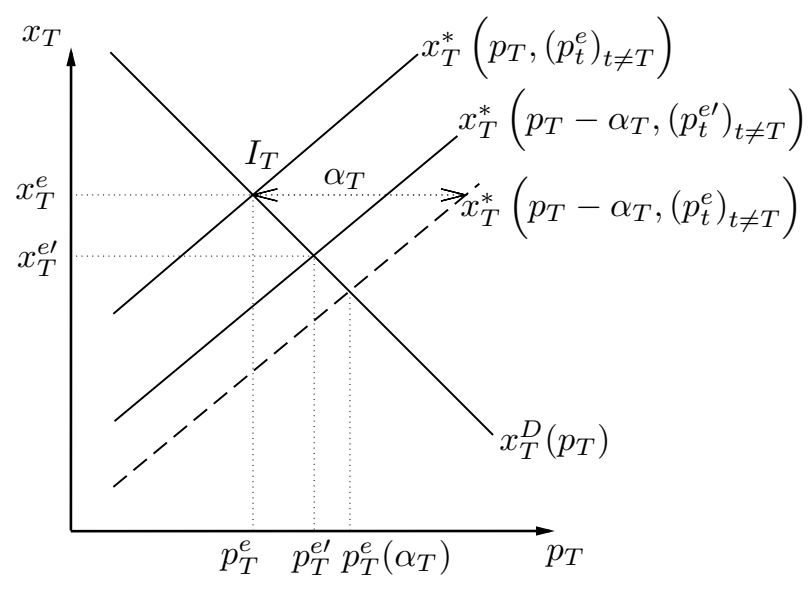

(a) Date $T$ of tax increase

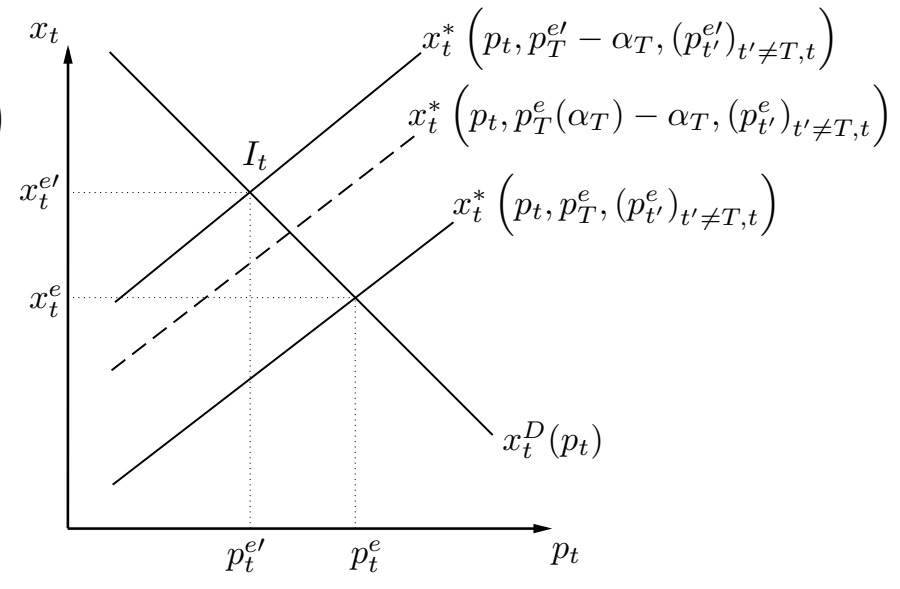

(b) Any date $t \neq T$

Figure 2: Partial-equilibrium effects of extraction taxation

occurring at date zero, consider the market at any particular date $t \geq 0$. For unchanged prices $p_{t^{\prime}}^{e}$ at dates $t^{\prime} \neq t$, date- $t$ supply is shifted down to $\widetilde{x}_{t}\left(p_{t},\left(p_{t^{\prime}}^{e}\right)_{t^{\prime} \neq t}, X^{\prime}\right)$, causing a rise in the equilibrium price. Since lower supplies at all other dates $t^{\prime} \neq t$ similarly increase all equilibrium prices $p_{t^{\prime}}^{e}$, supply curves at all dates are further shifted down. Fully adjusted equilibrium prices at all dates are $p_{t^{\prime}}^{e \prime} \geq p_{t^{\prime}}^{e}$, with equality if demand is infinitely elastic, and date- $t$ supply curve becomes $\widetilde{x}_{t}\left(p_{t},\left(p_{t^{\prime}}^{e \prime}\right)_{t^{\prime} \neq t}, X^{\prime}\right)$, lower than $\widetilde{x}_{t}\left(p_{t},\left(p_{t^{\prime}}^{e}\right)_{t^{\prime} \neq t}, X^{\prime}\right)$.

When reserves are endogenous, reserve reduction may be partly compensated by the development of new reserves. In that case, the supply of exploitable reserves $X(\lambda)$ defined by (6) is shifted down to $X(\lambda)-\Omega$. For a more general supply-reduction policy whose stringency is indexed by $\Omega$, the reserve supply function is redefined as $X(\lambda ; \Omega)$, with $\frac{\partial X}{\partial \lambda}>0$ and $\frac{\partial X}{\partial \Omega}<0 .{ }^{16}$ Intuition suggests, and Proposition 3 will confirm, that an increase in the policy's stringency from $\Omega$ to $\Omega^{\prime}>\Omega$ results in a greater equilibrium rent $\lambda^{e \prime}>\lambda^{e}$ and in lower equilibrium reserves $X^{e \prime}<X^{e}$. Thus whether the reserve reduction is compensated (endogenous reserves) or not (restricted reserves), instantaneous supplies are reduced at all dates, and the partial-equilibrium implications of a rise in $\Omega$ are qualitatively the same as those of a fall in $X$ described by Figure 3 .

\footnotetext{
${ }^{16}$ For example, suppose that exploitable reserves are reduced by an exogenous, policy induced, demand for reserves $X^{D}(\lambda ; \Omega)$, decreasing in $\lambda$ and increasing in the stringency index $\Omega$. Then, the reserve supply function becomes $X(\lambda ; \Omega) \equiv E^{\prime-1}(\lambda)-X^{D}(\lambda ; \Omega) \leq E^{\prime-1}(\lambda)$.
} 


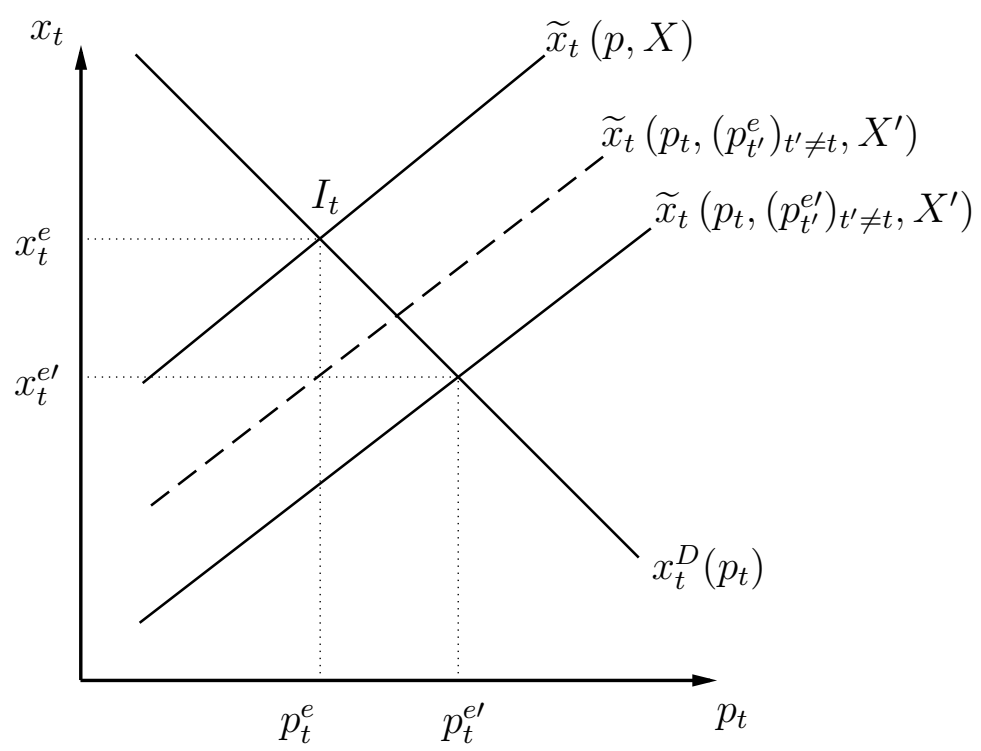

Figure 3: Partial-equilibrium effects of reserve reduction

Harstad addresses this problem first as a static problem; then in a two-period framework. He also considers that reserves come in different deposits of varying quality that are not in production when they are purchased. We deal with resource heterogeneity and endogenous production periods in Subsections 4.a and 4.b.

\section{3.c Demand-Reducing Policy}

Consider now a policy that reduces the demand for the NRR during the periods over which it is implemented. Assume that the demand $x_{t}^{D}$ for the resource at date $t \geq 0$ does not only depend on the date, on the resource price $p_{t}$ at that date, but also on the stringency at date $t$ of NRR demand-reducing policies, synthesized by the index $\theta_{t}$. NRR demand may thus be written ${ }^{17} x_{t}^{D}\left(p_{t} ; \theta_{t}\right)$ and assumed continuously differentiable and decreasing in both arguments, where $\theta_{t}=0$ means the absence of any demand-reducing policy. The path of the policy stringency index $\Theta \equiv\left(\theta_{t}\right)_{t \geq 0}$ is exogenously given.

Assume that a demand-reducing policy is implemented at date $T$ and only at that date, so the index $\theta_{T}$ rises from 0 to $\theta_{T}>0$ while $\theta_{t}=0, \forall t \neq T$; the partial-equilibrium

\footnotetext{
${ }^{17} \mathrm{An}$ increase in $\theta_{t}$ at any date $t$ may reflect policy-induced technical change, an increase in resource consumption taxation, an increase in subsidies to substitutes, or any combination of such resource demand-reducing policies.
} 
effect is depicted in Figure 4. Prices $p_{t}^{e}, t \geq 0$, denote equilibrium prices with $\theta_{T}=0$. When the policy is implemented, the demand at date $T$ shifts down. Initially assuming that prices at dates $t \neq T$ are unchanged, date- $T$ supply function does not change so that the drop in demand induces a move of date- $T$ equilibrium down along the supply curve. The producer price $p_{T}^{e}$ is reduced to $p_{T}^{e}\left(\theta_{T}\right)<p_{T}^{e}$; quantity $x_{T}^{e}$ is reduced accordingly (Figure 4(a)).

A lower producer price at $T$ causes the supply curves at all other dates $t \neq T$ to shift up by Proposition 1, resulting in a drop in price at these dates. In turn, reduced producer prices at dates $t^{\prime}$, also different from $T$, further reinforce the upward shift in the supply curve at $t$ (Figure $4(\mathrm{~b})$ ). Thus fully adjusted prices $p_{t}^{e \prime}$ are lower and quantities supplied are higher than before the policy implementation at all dates $t \neq T$. It follows that there is a feedback at $T$ which shifts supply up to $x_{T}^{*}\left(p_{T},\left(p_{t}^{e^{\prime}}\right)_{t \neq T}\right)$. This reinforces the initial drop in the price at $T$ while also causing an increase in quantity (Figure 4(a)). However, this quantity response only partially offsets the initial downward reduction in quantity along the supply curve. Indeed, when reserves are fixed as in the short-run, the increase in quantities at all dates $t \neq T$ implies that extraction must decrease at $T$; and in the long-run, as shown in Section 2, exploitable reserves are lowered by the demand-reducing policy, so that extraction at $T$ diminishes more than in the short run.

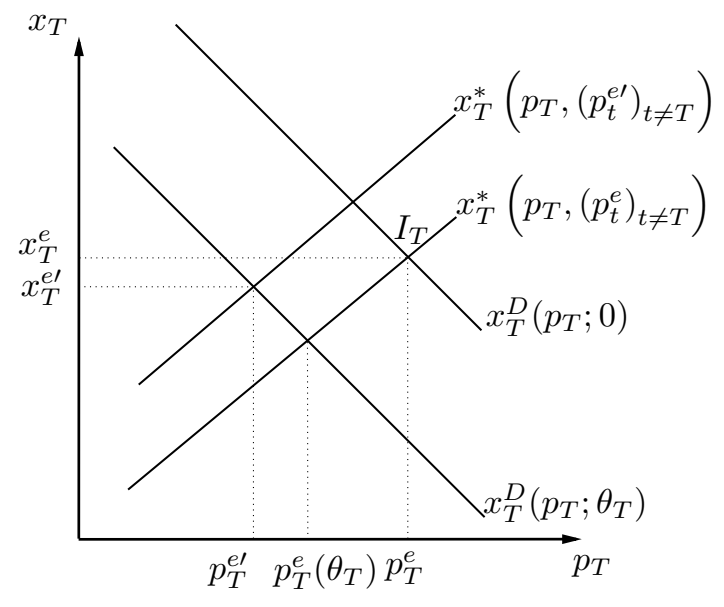

(a) Date $T$ of policy implementation

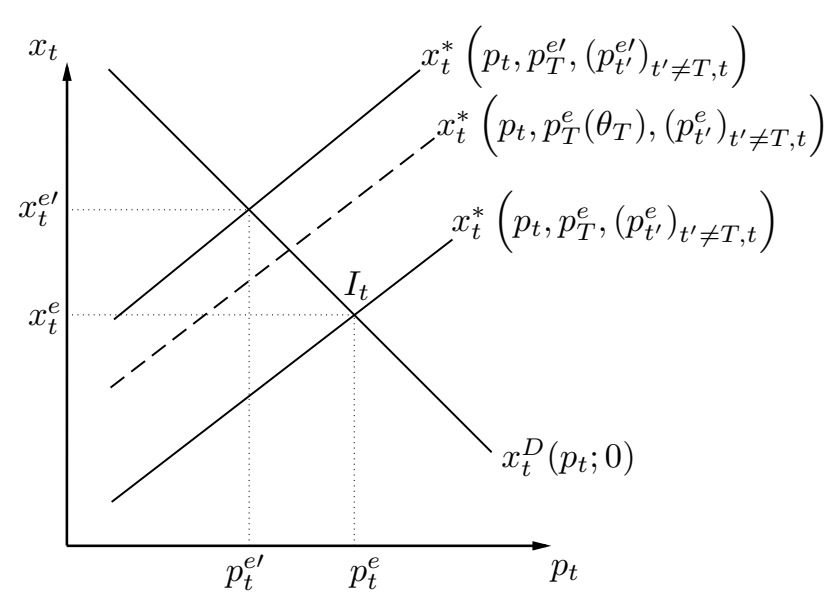

(b) Any date $t \neq T$

Figure 4: Partial-equilibrium effects of demand reduction 


\section{3.d Generic NRR Supply and Demand Policies}

The illustrations given above are simple examples. For more general applications, generic supply and demand policies can be modeled as follows; their effects are described in Proposition 3 further below.

A demand policy indexed by $\theta_{t}$ may reduce date- $t$ demand for the NRR to $x_{t}^{D}\left(p_{t} ; \theta_{t}\right)$ from its no-intervention level $x_{t}^{D}\left(p_{t} ; 0\right)$. Demand-reducing policies may take various forms, such as consumer taxes or support to NRR substitutes. Assuming that $x_{t}^{D}$ is continuously differentiable and monotonic in both arguments, the inverse demand function at $t$, $P_{t}\left(x_{t} ; \theta_{t}\right)$ is decreasing in $x_{t}$ and in $\theta_{t}$. In the sequel we will assume that stringency levels are chosen such that $P_{t}\left(0 ; \theta_{t}\right)$ is greater than the equilibrium price $p_{t}^{e}$ for some dates, to avoid situations where policies do not warrant any production at all.

A supply policy may aim at extraction while affecting reserves only indirectly; or it may focus on reserves directly. We will refer to the former as extraction policy, while calling the latter a reserve policy. An extraction policy indexed by $\xi_{t}$ reduces date$t$ marginal extraction profit $p_{t}-C_{t}^{\prime}\left(x_{t}\right)-\frac{\partial G_{t}\left(x_{t}, p_{t} ; \xi_{t}\right)}{\partial x_{t}}$, where the policy function $G_{t}$ is positive and such that the policy-adjusted cost function $C_{t}+G_{t}$ inherits the properties of the original cost function: it is increasing, strictly convex and twice differentiable in $x_{t}$; it also satisfies $C_{t}(0)+G_{t}\left(0, p_{t} ; \xi_{t}\right)=0$ for all $t \geq 0, p_{t}>0$ and $\xi_{t}$, and is such that $C_{t}^{\prime}(0)+\frac{\partial G_{t}\left(0, p_{t} ; \xi_{t}\right)}{\partial x_{t}}$ is lower than the equilibrium price $p_{t}^{e}$ for at least one date where $P_{t}\left(0 ; \theta_{t}\right)>p_{t}^{e}$ also holds. Last, it is assumed that $\frac{\partial^{2} G_{t}}{\partial x_{t} \partial p_{t}}<1$ to eliminate ill-conceived policies under which marginal cost would increase more than marginal revenue as a result of a price increase. We define a more stringent extraction policy as one that reduces the marginal extraction profit: $\frac{\partial^{2} G_{t}}{\partial x_{t} \partial \xi_{t}}>0, \forall x_{t} \geq 0$. Modified this way, Problem (1)-(2) yields necessary conditions

$$
x_{t}=x_{t}\left(p_{t}, \lambda ; \xi_{t}\right), \forall t \geq 0
$$

As their counterparts in (7), the new functions (23) are increasing in $p_{t}$ and decreasing in the rent $\lambda$; they are further decreasing in the extraction policy index $\xi_{t}$.

Next, a reserve-reducing policy increases the marginal cost of developing exploitable reserves. The exploration and development cost $E(X)$ in Problem (1)-(2) is augmented 
by the function $F(X ; \Omega)$, positive and increasing in its two arguments, where $\Omega$ reflects the stringency of the reserve-reducing policy. As in Section 2, assume that the total development cost $E(X)+F(X ; \Omega)$ is twice differentiable, strictly convex and satisfies $E(0)+F(0 ; \Omega)=E^{\prime}(0)+\frac{\partial F(0 ; \Omega)}{\partial X}=0$. Modifying Problem (1)-(2) accordingly, the necessary reserve-supply condition (5) is replaced by $E^{\prime}(X)+\frac{\partial F(X ; \Omega)}{\partial X}=\lambda$, which implicitly defines the policy-induced level of reserves as

$$
X=X(\lambda ; \Omega)
$$

As its counterpart (6), this function is increasing in the rent $\lambda$; it is further decreasing in the reserve-policy index $\Omega$. The NRR producer's supply behavior is summarized by the functions (23) and (24).

At this stage of the formalization in Section 2, the remaining step toward establishing supply functions was to replace the endogenous rent $\lambda$ by an appropriate function of parametric prices. In the current partial equilibrium analysis, prices are endogenous but one must recognize the dependency of $\lambda$ on both the supply and the demand policy parameters. This is done as follows.

For any date $t \geq 0$, substituting the inverse demand function $P_{t}\left(x_{t} ; \theta_{t}\right)$ into the supply function (23) implicitly defines date- $t$ equilibrium extraction as a function

$$
x_{t}=\widehat{x}_{t}^{e}\left(\lambda ; \xi_{t}, \theta_{t}\right), \forall t \geq 0
$$

This function is decreasing in the rent $\lambda$, in the extraction-policy index $\xi_{t}$, and in the demand-policy index $\theta_{t}$.

Treating the stock of initial reserves as given at this stage, and combining relations (25) at all dates into the exhaustibility constraint (2) defines the short-run equilibrium rent as a function $\widetilde{\lambda}^{e}(X ; \Xi, \Theta)$ of initial reserves, of the vector of extraction-policy indices $\Xi \equiv\left(\xi_{t}\right)_{t \geq 0}$, and of the vector of demand-policy indices $\Theta \equiv\left(\theta_{t}\right)_{t \geq 0}$. As its counterpart (8), $\tilde{\lambda}^{e}(X ; \Xi, \Theta)$ is decreasing in $X$; it can be shown that it is also decreasing in all elements of the policy vectors $\Xi$ and $\Theta$.

Substituting $\widetilde{\lambda}^{e}(X ; \Xi, \Theta)$ into each extraction function (25) gives the restricted (short- 
run) NRR equilibrium extraction functions:

$$
x_{t}=\widetilde{x}_{t}^{e}(X ; \Xi, \Theta) \equiv \widehat{x}_{t}^{e}\left(\widetilde{\lambda}^{e}(X ; \Xi, \Theta) ; \xi_{t}, \theta_{t}\right), \forall t \geq 0
$$

Each restricted NRR equilibrium extraction function is increasing in initial reserves $X$. Holding $X$ unchanged, the partial effects on equilibrium supply at $t$ of changing policy intensities $\xi_{T}$ and $\theta_{T}$ at any date $T \geq 0$ can be established as in Section 2's analysis of the effects of changing the price $p_{T}$ on restricted supply. The only difference is that a rise in $\xi_{T}$ or $\theta_{T}$ (a policy restriction) affects the quantity supplied in a direction opposite to that of a rise in price. ${ }^{18}$

Now consider the long term, allowing initial reserves to be endogenously determined. Substituting $\widetilde{\lambda}^{e}(X ; \Xi, \Theta)$ into (24), implicitly defines long-run equilibrium reserves

$$
X=X^{e}(\Xi, \Theta, \Omega)
$$

In turn, substituting $X^{e}(\Xi, \Theta, \Omega)$ into $\widetilde{\lambda}^{e}(X ; \Xi, \Theta)$ defines $\lambda=\lambda^{e}(\Xi, \Theta, \Omega) \equiv \widetilde{\lambda}^{e}\left(X^{e}(\Xi, \Theta, \Omega) ; \Xi, \Theta\right)$. It can be shown that $X^{e}$ is decreasing in $\xi_{t}$ and $\theta_{t}$, for all $t \geq 0$, as well as in $\Omega$, and that $\lambda^{e}$ is decreasing in $\xi_{t}$, in $\theta_{t}$, for all $t \geq 0$, but increasing in $\Omega$. The unrestricted (long-run) equilibrium extraction level at date $t$ is thus defined as the following function of all elements in the policy vectors $\Xi$ and $\Theta$ as well as the policy index $\Omega$ :

$$
x_{t}^{e}=x_{t}^{e}(\Xi, \Theta, \Omega) \equiv \widetilde{x}_{t}^{e}\left(X^{e}(\Xi, \Theta, \Omega) ; \Xi, \Theta\right)
$$

The short-run equilibrium extraction functions (26) and the long-run equilibrium extraction functions (28) just established have the same comparative static properties with respect to the exogenous (supply and demand) policy parameters, mutatis mutandis, as their NRR supply counterparts have with respect to the exogenous prices. This can be shown by adapting the steps followed in Section 2. In particular, the effects of supplyand demand-reducing policies on equilibrium extraction quantities can be decomposed

\footnotetext{
${ }^{18}$ For example consider an increase in $\theta_{T}$ at any date $T \geq 0$. By the definition (26) of the $\widetilde{x}_{t}^{e}$ restricted functions, extraction at all dates $t \neq T$ is only affected via the policy-induced change in the rent $\widetilde{\lambda}^{e}$ in $\widehat{x}_{t}^{e} ; \widetilde{\lambda}^{e}$ is reduced as a consequence of the rise in $\theta_{T}$, which in turn, by (25), increases $\widehat{x}_{t}^{e}$, for all $t \neq T$, and thus $\widetilde{x}_{t}^{e}$, for all $t \neq T$. Extraction being increased at all dates $t \neq T$ while exploited reserves are unchanged, equilibrium extraction must decrease at the date $T$ of the policy change. The same analysis applies to a change in the supply-policy stringency $\xi_{T}$.
} 
into a stock compensation effect and a pure substitution effect as illustrated by (12) in the case of the price effect in Section 2. Those properties are summarized in the following proposition.

\section{Proposition 3 (Policy-induced equilibrium changes on a single-outlet homogeneous- NRR market)}

1. A reserve-reducing policy decreases short-run and long-run equilibrium extraction at all dates $t \geq 0$.

2. Any combination of extraction and demand-reducing policies at any date $T$

(a) (Stock effect) reduces long-run developed reserves;

(b) (Contemporary-price effect) reduces short-run and long-run extraction at date $T$;

(c) (Cross-price effects) increases short-run and long-run extraction at all dates $t \neq T$.

Proposition 3 focuses on the equilibrium of a single-outlet homogeneous-NRR market. It can be readily extended to multiple-outlet markets using the formalization leading to Proposition 2. ${ }^{19}$

\section{3.e Policy Effects over Extended Periods and Areas}

The results stated in Proposition 3 can be used to study the effects of policies that reduce NRR demand and/or supply over some extended future period and in specific locations via various forms of restrictions to NRR extraction and use, or assistance to alternative sources of supply.

Consider an increase in policy stringency at several dates $T$ that form a set $\Delta$. Proposition 3.2 establishes that such increases in policy stringency have the same qualitative effect on equilibrium NRR quantities at dates when no policies are implemented. Thus all effects combine to positively affect extraction at all dates $t \notin \Delta$.

\footnotetext{
${ }^{19} \mathrm{~A}$ proof is available from the authors upon request.
} 
When policies are unanticipated and not accompanied by any adjustment in the stock of reserves, they affect the restricted equilibrium supply $\widetilde{x}_{t}^{e}(X ; \Xi, \Theta)$; when they are anticipated and associated with a drop in developed reserves, they affect the unrestricted equilibrium supply $x_{t}^{e}(\Xi, \Theta, \Omega)$. In either case, reductions for example in demand at $T \in \Delta$ increase extraction at all $t \notin \Delta$, confirming the validity of the green paradox.

If the policy change occurs at a single date as in Proposition 3.2, the drop in price unambiguously causes a drop in extraction at that date. When $\Delta$ contains more than a single date, the reaction of NRR extraction at each $T \in \Delta$ depends on the magnitude of the price change occurring at that date relative to the changes occurring at other dates $T^{\prime} \in \Delta$. However, the above analysis indicates that cumulative extraction over $\Delta$ is reduced. This is because $X$ decreases while cumulative supply at all dates $t \notin \Delta$ increases.

\section{Corollary 1 (Policy-induced equilibrium changes over extended periods)}

An extraction or demand-reducing policy implemented at dates $T \in \Delta$

1. (Stock effect) reduces long-run developed reserves;

2. (Contemporary-price effect) reduces short-run and long-run cumulative extraction over dates $T \in \Delta$;

3. (Cross-price effects) increases short-run and long-run extraction at all dates $t \notin \Delta$.

As suggested already, extraction leakages (Fischer and Salant, 2013; Gaudet, Moreaux and Salant, 2001) work in the same direction spacewise, and timewise, in the short run and in the long run. ${ }^{20}$ When the change in demand affects more than one region as with Fischer and Salant's (2013) technology-oriented policies, or takes place at more than one date as with their emission taxes, the reaction of resource supply to one region at one date depends on the magnitude of the price change occurring in that region and date relative to the changes occurring at other regions and dates. At dates and regions not concerned by the policies, short-run and long-run supply increases.

\footnotetext{
${ }^{20}$ The spatial version of Corollary 1 can be established using the formalization of Proposition 2; a proof is available from the authors on request.
} 


\section{3.f Market Power}

A firm that enjoys market power on the NRR market solves Problem (1)-(2) while taking into account the incidence on prices of its quantity decisions: marginal profits on the left-hand side of (4) become, instead of $p_{t}-C_{t}^{\prime}\left(x_{t}\right), p_{t}-x_{t} \frac{d p_{t}}{d x_{t}}-C_{t}^{\prime}\left(x_{t}\right)$. Although prices are no longer exogenous, they are affected by exogenous policy parameters in a known way. Hence policies may be modeled as in Subsection 3.d.

On the production side, the treatment is unchanged. A short-run extraction policy indexed by $\xi_{t}$ raises date- $t$ marginal extraction cost to $C_{t}^{\prime}\left(x_{t}\right)+\frac{\partial G_{t}\left(x_{t}, p_{t} ; \xi_{t}\right)}{\partial x_{t}}$, where the policy-adjusted cost function $C_{t}+G_{t}$ exhibits the properties established in Subsection 3.d; and similarly, the exploration and development cost $E(X)$ is augmented by the function $F(X ; \Omega)$ as above.

On the demand side, policy parameters $\theta_{t}$ reduce the inverse demand for the NRR at $t$ from the no-intervention level $P_{t}\left(x_{t} ; 0\right)$ to $P_{t}\left(x_{t} ; \theta_{t}\right)$. The difference with the competitive case treated in Subsection 3.d is that a meaningful demand policy must not only be such

that $P_{t}\left(x_{t} ; \theta_{t}\right)$ is decreasing in $\theta_{t}$ but such that the marginal revenue $\frac{\partial P_{t}\left(x_{t} ; \theta_{t}\right) x_{t}}{\partial x_{t}}$ exhibits the same property. For equilibrium supplies to be well defined, the analysis further requires that net revenue $P_{t}\left(x_{t} ; \theta_{t}\right) x_{t}-C_{t}\left(x_{t}\right)-G_{t}\left(x_{t}, p_{t} ; \xi_{t}\right)$ be strictly concave in $x_{t}$.

Provided these conditions are satisfied, the impact of policies on short-run and longrun extraction and on the stock of developed reserves is thus determined by functions with the same properties as (26), (27) and (28) when the producer has market power. In other words, Proposition 3 applies with no further restriction than the above concavity in $x_{t}$ of profits.

\section{Heterogenous Resources}

Non-renewable resources are notoriously heterogeneous. This heterogeneity is mostly manifest in the costs involved in discovering, developing, and extracting the resource, as well as transforming it into a homogenous commodity. The seminal intuition of Herfindahl (1967) known as the "least-cost-first" principle has given rise to a substantial, sometimes 
controversial, literature. ${ }^{21}$

One strand of literature considers that industry NRR supply consists of contributions from individual deposits and studies the sequence of their exploitation. It stems directly from Herfindahl's initial contribution showing that deposits of constant but different unit costs of extraction were to be exploited in a strict sequence. As was later established, simultaneous exploitation of different deposits may be optimal when marginal costs depend on extraction rates or capacities are limited or costly to acquire (see, e.g. Amigues et al., 1998; Gaudet and Lasserre, 2011; Salant, 2013). The least-cost-first principle may further fail, most notably when deposits differ in size. In this multi-deposit approach, resources are homogenous within a deposit and heterogeneity results from differences between deposits.

An alternative way to model NRR heterogeneity is due to Hotelling (1931, p. 152), ${ }^{22}$ has been consolidated by Gordon (1967), further discussed or refined by Weitzman (1976), Levhari and Liviatan (1977), Pindyck (1978), Salant et al. (1983) and Sweeney (1993); it was used recently by Gerlagh (2011), Hoel (2012), van der Ploeg and Withagen (2012a, 2012b, 2014), Grafton et al. (2012), and Golosov et al. (2014) in works related to resource taxation and the green paradox. By assumption, the cost of extraction increases with cumulative extraction under the rationale that least-cost resources are used first irrespective of the deposit from which they are extracted. Reserves may be left underground if and when the unit extraction cost is not met by the price, which implies that prices affect the level of economic reserves. Although the original paper of Gordon argues that the increase in extraction costs with cumulative extraction occurs at the firm level as well as in the aggregate, Gordon's postulate is often implicitly assumed to apply across deposits at the industry level implying that the relevant stock of reserves is the industry aggregate

\footnotetext{
${ }^{21}$ As Slade (1988) puts it "The idea that the least-cost deposits will be extracted first is so firmly embedded in our minds that it is an often-made but rarely tested assumption underlying the construction of theoretical exhaustible-resource models." (p. 189). The particular notion of cost involved and context of analysis are further subject to appreciation (Kemp and Long, 1980). Are the costs inclusive of resource rents or not (Hartwick, 1978; Amigues and Moreaux, 2002)? Are the relevant costs short-run costs or long-run costs (Hartwick et al., 1986)? What variables, extraction rates and reserve levels most importantly, determine extraction costs? Does an industry cost function exist or should costs be measured at the deposit level?

${ }^{22}$ Although Hotelling is not usually credited for it.
} 
stock and that the corresponding cost function is an aggregate industry cost function. ${ }^{23}$

We show how the properties of NRR supply established in Section 2 carry over when the resource is heterogenous, whether heterogeneity takes the form of inter-deposit heterogeneity as in the branch of literature that stems from Herfindahl's initial paper, or takes the form of intra-deposit heterogeneity as in the tradition initiated by Gordon. Consider the latter first, in the next subsection; we will deal with multiple deposits in a subsequent subsection, where we also make opening dates endogenous.

\section{4.a Hotelling-Gordon Model: Stock Effects in Extraction Costs}

Since the resource is not homogenous, it is important to make a distinction between geological and economical reserves. Let $X_{t}$ represent the stock of geological reserves remaining at $t$, and relabel the stock of initial geological reserves $X^{0}$, with $X_{0}=X^{0}$. Units of measurement are chosen such that the flow of extraction is homogenous over time, e.g. barrels of oil of constant energy content, viscosity, refining cost, etc. Cumulative extraction between dates 0 and $t$ is equal to $X_{0}-X_{t}$, with

$$
X_{t+1}=X_{t}-x_{t}, X_{0}=X^{0} \text { given }
$$

The extraction cost function may be written as $C_{t}\left(x_{t}, X_{t}\right)$. Under such a technogeological constraint on extraction, the net present-value extraction revenue $p_{t} x_{t}-C_{t}\left(x_{t}, X_{t}\right)$ not only depends on $x_{t}$ and $p_{t}$ as in Section 2, but also on remaining reserves $X_{t}$. It is still true at each date $t$ that $\frac{\partial C_{t}(x, X)}{\partial x}>0$ and $C_{t}(0, X)=0, \forall X \geq 0$. Also, we assume that $\frac{\partial C_{t}\left(0, X^{0}\right)}{\partial x}<p_{t}$ for at least one date so that some exploitation is warranted. The dependency of extraction cost on cumulative extraction implies that the cost depends on $X$ negatively: higher current reserves imply lower cumulative extraction, hence a lower cost. We assume

$$
\frac{\partial C_{t}(x, X)}{\partial X}<0 \text { and } \frac{\partial C_{t}(x, X)}{\partial x \partial X}<0
$$

for all $t \geq 0$ and for all $x>0$ and $X \geq 0$.

\footnotetext{
${ }^{23}$ A related modeling approach has its roots in the energy literature and finds its most recent expression in Venables (2014). Resources not only differ in their cost of extraction, but the ultimate amount that may be recovered at a cost below some specified cut-off level may depend on the speed at which extraction proceeds.
} 
We also assume that $C_{t}(x, X)$ is strictly convex in its two arguments. ${ }^{24}$ We owe James Sweeney (1993) a thorough investigation of the discrete version of the Hotelling-Gordon model; as he showed, the existence of an underlying continuous-time representation of the technology implies restrictions on the partial derivatives of allowable discrete-time cost functions; precisely, it must be true that

$$
\frac{\partial^{2} C_{t}(x, X)}{\partial x^{2}}+\frac{\partial^{2} C_{t}(x, X)}{\partial x \partial X}>0
$$

a property called the dominance of extraction rate on marginal $\operatorname{cost}^{25}$ which will also be assumed to hold here. ${ }^{26}$

As in the simple model of Section 2, the producer must identify and develop the reserves to be exploited before extraction begins. A portion $X^{0}-X^{F}$ is chosen within the initial stock $X^{0}$ of geological reserves and undergoes a costly exploration and development process that makes it suitable for exploitation. No development expenditure is applied to reserves that are not deemed economical, implying that the stock $X^{F} \geq 0$ of geological reserves left undeveloped will be left unexploited at the end of the extraction process.

The amount $X^{0}-X^{F}$ defines economic reserves. Economic reserves can be increased at date zero by reducing $X^{F}$. It is sensible to assume decreasing returns to exploration and development on the ground, as argued before, that the best prospects are developed first. Redefining the function $E$, we thus assume that the cost of developing an initial stock of exploitable economic reserves $X^{0}-X^{F}$ when geological reserves are $X^{0}$ is $E\left(X^{0}-X^{F}\right),{ }^{27}$

\footnotetext{
${ }^{24}$ Under the maintained assumption that the cost function is convex, the objective function for this problem is concave and the feasible set is convex. Thus the first-order necessary conditions are sufficient for optimality and multiple local unconnected optima cannot exist (Sweeney, 1993, p. 771). We further assume the strict convexity of the cost function in order to avoid having to deal with supply correspondences rather than supply functions.

${ }^{25}$ Salant et al. (1983) also imposed this assumption, although not in reference with any underlying continuous-time technology.

${ }^{26}$ As a simple way to focus on the rise in extraction cost with cumulative extraction, the extraction cost of the underlying continuous-time cost function is sometimes assumed to depend on the remaining-reserve stock, but not on the extraction rate (e.g. van der Ploeg and Withagen, 2012a among many others); i.e. total cost is assumed linear in extraction rate. However, this linearity assumption in a continuous-time model implies a discrete-time representation in which marginal extraction cost is a strictly increasing function of extraction rate and a decreasing function of the remaining stock (Sweeney, 1993). Thus the discrete-time model presented here encompasses both continuous-time versions of Gordon's model that treat the marginal cost of extraction as constant or as strictly rising.

${ }^{27}$ We write the amount of reserves developed for exploitation as $X^{0}-X^{F}$ rather than merely $X$ as
} 
with $E(0)=0$ and $E^{\prime}(0)=0 .{ }^{28}$

The problem faced by a NRR producer under such conditions is (see the Appendix for details of the resolution)

$$
\max _{\left(x_{t}, X_{t}\right)_{t \geq 0}} \sum_{t \geq 0}\left(p_{t} x_{t}-C_{t}\left(x_{t}, X_{t}\right)\right)-E\left(X^{0}-X^{F}\right)
$$

subject to

$$
\begin{gathered}
X_{t+1}=X_{t}-x_{t}, \forall t \geq 0, \\
x_{t} \geq 0, \forall t \geq 0, \\
X_{0}=X^{0} \text { given } \\
X_{t} \geq X^{F} \geq 0, \forall t \geq 0,
\end{gathered}
$$

where economic reserves $X^{0}-X^{F}$, and thus also geological reserves $X^{F}$ left undeveloped at the end of exploitation, are fixed in the short run but endogenous in the long run.

We denote by $S$, which may be infinite, the last date at which strictly positive extraction occurs. Thus if $S$ exists, $X_{t}=X_{t+1}$ for all $t \geq S+1$. Clearly, extraction may also be null occasionally before $S$.

Because of resource heterogeneity, the present-value resource rent measured by the Lagrangian multiplier associated with (32) is not constant over time. It is sometimes called a Ricardian resource rent and diminishes as reserves diminish; we denote it $\mu_{t}$ rather than $\lambda$, the Lagrangian multiplier associated with the same constraint in Section 2, to emphasize that this rent is different from a pure Hotelling scarcity rent.

Pure scarcity arises in this model at two levels, associated with the two inequality constraints in (35). The right-hand side inequality addresses the possibility that the

in Section 2 in order to emphasize an important property of the model. The marginal unit of reserves being developed at date zero is the unit that will be extracted last, not first. In models of homogenous resources, this does not matter; in Gordon's model, the sequence of reserve development and extraction is a geological and technological assumption, although it is often justified on economic grounds. Increasing the stock of developed reserves at date zero means reducing the amount of geological reserves $X^{F}$ to be left unexploited at the closure date. As a result, the cost of extraction at date zero is the same whatever the amount of developed reserves.

${ }^{28} \mathrm{As}$ in Section 2, the property $E^{\prime}(0)=0$ is introduced because it is sufficient to ensure that a positive amount of reserves is developed. It plays no other role than ruling out uninteresting situations where resource prices do not warrant the production of any reserves. 
totality of geological reserves be worth exploiting. It has been well investigated (e.g. Levhari and Liviatan, 1977) and will be considered only in the Appendix. The lefthand side inequality recognizes the assumption that only those reserves that have been previously discovered and developed at date zero may be exploited. As already argued, exploration and reserve development are costly, so that no reserves are developed to be ultimately left unexploited. Prior to the exhaustion of economic reserves, $X_{t}>X^{F}$ and the constraint is not binding; at the date of exhaustion, the constraint becomes binding and we denote the associated multiplier by $\lambda$ with no time index because the date of exhaustion $S$ is endogenous; $\lambda$ may be interpreted as the pure Hotelling component of the resource rent in a short-run perspective, or, in a long-run perspective, as a quasirent reflecting both pure Hotelling scarcity and expenditures sunk in exploration and development.

The first-order condition for strictly positive extraction at date $t$ is

$$
p_{t}-\frac{\partial C_{t}\left(x_{t}, X_{t}\right)}{\partial x}=\mu_{t}, x_{t}>0, \forall t=0, \ldots, S
$$

The resource rent evolves according to the first-order condition

$$
\begin{aligned}
\mu_{t}= & \mu_{t-1}+\frac{\partial C_{t}\left(x_{t}, X_{t}\right)}{\partial X}, \forall t=0, \ldots, S, \\
& \text { with } \\
\mu_{S}= & \lambda \text { and } \lambda=E^{\prime}\left(X^{0}-X^{F}\right) \text { in the long run if } X^{F}>0 .
\end{aligned}
$$

According to (37), the present-value rent diminishes as the stock of reserves diminishes and extraction cost increases. At the end of operations, all pre-developed reserves are exhausted so that remaining geological reserves equal $X^{F}$. When those remaining reserves are not null, the constraint that $X^{F} \geq 0$ is not binding and the resource rent equals the cost of finding and developing the marginal unit of economic reserves, as stated by the right-hand equality in (38).

The following proposition characterizes the effect on restricted supply of a change in restricted reserves, and the effect on restricted and unrestricted supply at any date of a change in price occurring at any other date of the exploitation phase. They are 
the counterpart, in the context of the Hotelling-Gordon model, of Proposition 1 which applies to a homogenous resource. As in Proposition 1, intertemporal cross-price effects are negative, whether reserves are endogenous or not; the impact of a price change on reserves does not dominate the direct substitution effect. However, this result holds in terms of cumulative, rather than instantaneous, supply. ${ }^{29}$

\section{Proposition 4 (Supply from a heterogeneous NRR deposit)}

1. Stock effect: An exogenous rise in exploitable reserves increases short-run cumulative supply at all dates.

2. Cross-price effects: A price rise at any date $T \leq S$ reduces short-run and long-run cumulative supply at all dates $t \neq T$, where cumulative supply if $t>T$ is defined to exclude the supply at date $T$.

Proposition 4 concerns the individual deposit. The model with stock effects is also widely used at the aggregate level which raises several issues. For instance, the consistency of the Hotelling-Gordon approach at the aggregate level requires the existence of an aggregate reserve stock. ${ }^{30}$

In fact resource heterogeneity is manifest in differences between deposits - differences in size, exploration and development costs, and extraction costs mostly - probably more than in within-deposit differences. In the next section, we deal with multiple deposits that differ from one another, but are individually homogenous as in Section 2. Both the date at which they enter into production and the size of the reserves at which they do so are endogenous. The multiplicity of heterogenous sources requires that supply be examined both at the level of the individual deposit and in the aggregate.

\footnotetext{
${ }^{29}$ Thus the results of Section 2 hold in terms of cumulative supply when extraction costs change with the stock of remaining reserves. They hold in terms of flows at date zero, the current date of extraction of the planning horizon. Indeed, from the present perspective of the producer, current supply and cumulative supply are identical.

${ }^{30}$ According to Livernois and Uhler (1987)" if the sign of the relationship between the aggregate reserve base and aggregate extraction costs is ambiguous, then these models have very little to predict about the nature of optimal exploration or the likely shape of price paths."
} 


\section{4.b Multiple Deposits with Endogenous Production Periods}

As just argued, it is preferable to construct aggregate NRR supply as the sum of supplies from distinct individual deposits. We do so in this section, where we consider a multiplicity of different deposits identified by $j$, developed at endogenous dates $\tau^{j}$, and contributing to the supply of a unique resource whose price is $p_{t}$. Each deposit is similar to the single deposit considered in Section 2. However deposits differ by their size $X^{j}$, their geology, location and depth or quality, as reflected in the technologies underlying both extraction costs $C_{t}^{j}$ and exploration costs $E_{t}^{j}$ as well as in the evolution of these technologies over time. Were opening dates exogenous as in Section 2, Proposition 1 would imply that current supply diminishes as a result of a rise in price at any future date $T>0$, for each deposit already developed, hence also at the aggregate level.

There are various possible supply sources $j=1, \ldots, J$ (deposits, developed or not) that may contribute to resource supply. At date $t \geq 0$, let aggregate resource supply be

$$
S_{t}=\sum_{j=1}^{J} x_{t}^{j}
$$

where $x_{t}^{j} \geq 0$ is the current supply of resource $j$. Net spot extraction revenues are $p_{t} x_{t}^{j}-C_{t}^{j}\left(x_{t}^{j}\right)$, where the cost $C_{t}^{j}$ has the same properties as in the single-source case of Section 2. Since $x_{t}^{j}$ may be zero, there is no loss of generality in assuming that the same set of dates applies for all sources. Each source is constrained by its own finite reserve stock. As the marginal reserve unit of any deposit will only be developed if it is to be exploited, that constraint binds: ${ }^{31}$

$$
\sum_{t \geq 0} x_{t}^{j}=X^{j}, j=1, \ldots, J
$$

Each source is characterized by its own exploration and development cost $E_{t}^{j}\left(X^{j}\right)$, expressed in present value, whose qualitative properties are the same as in the case of a single resource, with the following minor difference. The property $E_{t}^{j \prime}(0)=0$ for some appropriate $t$ is only assumed for one deposit to ensure that some reserves production

\footnotetext{
${ }^{31}$ If deposit $j$ is never developed, its developed reserves are trivially zero so that $\sum_{t>0} x_{t}^{j}=0=X^{j}$.
} 
is warranted; otherwise $E_{t}^{j}{ }^{\prime}(0) \geq 0$, so that a resource whose marginal exploration and development cost is too high for profitability need not be developed. However the same deposit that is not economic at some early date may be developed when prices become higher or when the technologies encompassed in the functions $C_{t}^{j}$ and $E_{t}^{j}$ justify it. ${ }^{32}$ We assume that technological progress on exploration and development is such that, for any date $t^{\prime}>t$ and initial reserves $X^{j} \geq 0$,

$$
E_{t}^{j}\left(X^{j}\right) \geq E_{t^{\prime}}^{j}\left(X^{j}\right) \text { and } E_{t}^{j \prime}\left(X^{j}\right) \geq E_{t^{\prime}}^{j \prime}\left(X^{j}\right), \forall j
$$

As before, it is supposed that exploration and development are instantaneous and undertaken only once for each deposit; extraction may take place only after deposit development. All potential producers face the same given known price stream. For source $j$, the producer solves

$$
\max _{\left(x_{t}^{j}\right)_{t \geq 0}, X^{j}, \tau^{j}} \sum_{t \geq 0}\left(p_{t} x_{t}^{j}-C_{t}^{j}\left(x_{t}^{j}\right)\right)-E_{\tau^{j}}^{j}\left(X^{j}\right)
$$

subject to (40) and to

$$
x_{t}^{j}=0, t<\tau^{j}
$$

The development date $\tau^{j} \geq 0$ for deposit $j$ is free. Suppose that $\tau^{j}>0$. No production occurs before that date, so that $x_{t}^{j}=0, t<\tau^{j}$. We further assume that the evolution over time of resource price changes and extraction technology is such that, once initiated, production is not interrupted until exhaustion. ${ }^{33}$ We also assume that the problem is well behaved in the sense that the optima being characterized are global rather than local, at least in the neighborhood of the price vector under consideration. This rules out jumps from one local maximum to another local maximum as a result of a small change in the price vector. Clearly, the problems to be solved for each supply source are independent of each other. Thus the sole difference with the one-resource case analyzed in Section 2

\footnotetext{
${ }^{32}$ For some price and technology combinations, development occurs only at $\tau=0$ if at all. Such is the case, for example, if (present-value) prices are non increasing while (present-value) extraction and development costs are non decreasing.

${ }^{33}$ This assumption facilitates the analysis while it eliminates situations of only minor economic interest such as temporary interruptions of production. It is satisfied if prices do not diminish too fast and technological change is such that extraction costs do not increase too fast over any part of the exploitation period.
} 
is the fact that all deposits need not be developed at date zero if at all. Roughly, given a price path, resources whose extraction cost is higher and/or whose cost of exploration and development is higher will be developed later. We are interested in aggregate resource supply at dates $t \geq 0$; in particular we want to determine how aggregate supply $S_{t}$ reacts to a change in price at $T \geq t$. Since each component $x_{t}^{j}$ of $S_{t}$ is determined independently of the others, consider deposit $j$ in particular.

Supply from deposit $j$ may be restricted in two ways. First, as in Section 2, the size of reserves brought into production may be treated as exogenous; second the timing of deposit development may be treated as exogenous. While conceptually any combination of these two restrictions may apply, it seems to make more sense to consider that they apply in a specific order: in the very long run, no restriction; in the medium run, the timing of development is restricted but the size of reserves is endogenous; in the short run both the development date and the size of reserves are given.

Suppose at this stage that the development date $\tau^{j}$ is given (short/medium run). Then the derivations and properties established in Section 2 for Problem (1)-(2) can be readily adapted to Problem (42)-(40)-(43). There is one specific resource rent $\lambda^{j}$ associated with each deposit. If $\tau^{j}=0$, the solution is identical to that of Section 2 ; if $\tau^{j}>0, x_{t}^{j}=0$ when $t<\tau^{j}$ and, for $t \geq \tau^{j}$, the first-order conditions for the choice of the optimum extraction path and initial reserves differ from (4) and (5) only by the inclusion of time and deposit indexes:

$$
\begin{gathered}
p_{t}-C_{t}^{j \prime}\left(x_{t}^{j}\right)=\lambda^{j}, \forall t \geq \tau^{j} ; \\
E_{\tau^{j}}^{j \prime}\left(X^{j}\right)=\lambda^{j}
\end{gathered}
$$

All properties of the supply functions established in Section 2 apply for each deposit, provided it is active at $t$.

Still holding $\tau^{j}$ constant, $j=1, \ldots, J$, consider the effect of an increase in price at date $T$ on date- $t$ industry supply. All deposits developed at or before $t$ contribute to $S_{t}$. In the medium run, when the size of reserves in these deposits is a choice variable, all results from Proposition 1.2 on unrestricted supply are valid. The effect on aggregate supply at date $t$ is the sum of the changes in the supply from all deposits such that $\tau^{j} \leq t$ : if $t \neq T$, 
an increase in $p_{T}$ reduces extraction from all active deposits at $t$ reducing total supply at $t$. If $t=T$, a rise in $p_{T}$ increases the contribution from all deposits, thus increasing total supply at $t$.

Similarly in the short run, when both the size of exploitable reserves $X^{j}$ and the date of their development $\tau^{j}$ are fixed, the properties of restricted supply stated in Proposition 1.1 and .2 apply. A greater stock of exploitable reserves in a deposit positively affects supply from this deposit at all dates $t \geq \tau^{j}$; an increase in $p_{T}$ affects supply, at the deposit and at the aggregate level, in the same direction as above for the medium run. These results are gathered further below in Proposition 5.

In the long run, development dates $\tau^{j}$ are allowed to adjust to a price change: producers not only control their reserve development efforts, but also the date at which those efforts are made. The optimal choice of $\tau^{j}$ by the producer of deposit $j$ is denoted $\tau^{j *}(p)$. The optimal development date of deposit $j$ may be the corner solution $\tau^{j *}=0$, or, if it is an interior solution, $\tau^{j *}$ is a non-zero integer within the set of possible dates. The Appendix establishes the following lemma about the effect of a change in price on deposit-opening dates.

\section{Lemma 1 (Endogenous opening date of a NRR deposit)}

A price rise at any date after the planned opening of a deposit either leaves the opening date of the deposit unchanged, or postpones it to a date closer and anterior to the date of the price rise;

A price rise at any date prior to the planned opening of a deposit either leaves the opening date of the deposit unchanged, or accelerates it.

Consider a price rise at $T>0$. Lemma 1 indicates that all deposits that were active before $T$ have unchanged or postponed development dates. Clearly for deposits with unchanged development date the results just described for the medium run (developmentdate restricted) carry over to the long run (unrestricted development date) so that their combined production diminishes. For deposits whose development is postponed their production becomes zero until the new development date, which is inferior or equal to $T$. However it cannot be ruled out that their production in the new program at dates 
following the new development date might exceed their production at those dates under the initial price. Consequently, it is certain that aggregate production diminishes from date zero until the date of the first postponed deposit development if any. ${ }^{34}$ In particular, a price rise at any date $t>0$ always reduces aggregate supply at the present extraction date $t=0 .{ }^{35}$ These findings are gathered in the following proposition.

\section{Proposition 5 (Aggregate supply from multiple heterogeneous NRR deposits)}

1. Stock effect: An exogenous rise in exploitable reserves increases short-run (reserverestricted) aggregate supply at all dates.

2. Cross-price effects on short- and medium-run supply: A price rise at any date T reduces short-run (reserve and development-date restricted) aggregate supply and medium-run (development-date restricted) aggregate supply at all dates $t \neq T$.

3. Cross-price effects on long-run supply: A price rise at any date $T>0$ reduces longrun (unrestricted) aggregate supply at all dates $t \neq T$ of an initial period that extends at least from date zero until the date of the first postponed deposit development. If the functions $E_{t}^{j \prime}$ are time autonomous, a price rise at $T>0$ reduces long-run aggregate supply at all dates $t \neq T$.

\section{Conclusion}

The supply of commodities that must be produced before being dispatched over time and space differs from conventional supply in that supply functions then depend on a vector of parametric prices rather than a single price. The inventory to be dispatched or, in the case of NRR supply, the reserves to be extracted, may be given. In that case supply consists in optimally allocating a given stock (inventory or reserves) over time and space.

\footnotetext{
${ }^{34}$ Formally, a price rise at $T>0$ reduces supply from deposit $j$ at all dates $t \neq T$ if $j$ is active at $t$ in the initial program $\left(t \geq \tau^{j *}(p)\right.$, where $p$ is the initial price vector) in the two following cases:

1) the development date of the deposit is the same in the initial and new programs $\left(\tau^{j *}(p)=\tau^{j *}\left(p^{\prime}\right)\right.$, where $p^{\prime}$ is the new price vector), implying that the deposit is still active at $t$ after the price rise;

$2)$ the deposit is inactive at $t$ in the new program $\left(\tau^{j *}\left(p^{\prime}\right)>t \geq \tau^{j *}(p)\right)$.

${ }^{35}$ In the Appendix we show that if the marginal cost of development functions $E_{t}^{j \prime}$ are time autonomous, then the restriction to the date of the first postponed deposit development no longer applies.
} 
The supply functions, called restricted because they are conditional on the given factor, are then functions of the parametric prices prevailing at all dates and locations, as well as the quantity of the restricted factor. This defines the short run. In the long run, the stock of reserves (or the production inventory) is chosen endogenously at the beginning of the exploitation period so that the (unrestricted) supply functions depend on prices only. The beginning of the exploitation period may itself be endogenous, and we have shown how this impacts aggregate supply.

While the commodities supplied in fine are homogenous across the paper, the restricted factor has been allowed to be alternatively homogeneous or heterogeneous. This is important in general and especially in the case of NRRs. For instance, NRR reserves may vary in quality, accessibility, cost of discovery and development, etc. This is also often important in the case of non-resource commodities as conditions of production may vary according to origin or due to technological change and other factors. The focus on NRRs stresses the mechanisms at work in presence of heterogeneity and the resulting supply properties.

The law of supply is only one property of the supply functions that we have characterized. This paper has focused on cross-price effects: the effect on supply at one date and location of changes in prices at other dates or locations. They were decomposed into substitution effects (across time or space) and long-run stock compensation effects.

The substitution effect (across time or space) is always negative. When a NRR is homogenous, it dominates the long-run stock compensation effect, which is positive. Consequently both the short-run and the long-run cross-price effects are negative.

Besides filling a gap in the analysis of supply functions, this result confirms or provides several policy results. We give the examples of resource taxation, supply control policies, the green paradox, and leakage, giving generality to results usually discussed on the basis of particular cases.

More importantly, the highly-orthodox theoretical apparatus developed in the paper extends the conventional treatment of supply to commodities whose supply is determined across time and space according to parametric prices defined at dates and locations. By so 
doing, the paper extends to such supply situations the tool of partial equilibrium analysis and the familiar method of assessing the effects of policies by analyzing shifts in demand and supply curves.

In order to address NRR heterogeneity, we have relied on the two main approaches found in the resource literature. When the stock of reserves affects extraction costs so that there is intra-deposit heterogeneity, we find that the substitution effect again dominates the stock compensation effect, though this result must be expressed in terms of cumulative supply rather than flows. When heterogeneity is an inter-deposit feature, its effect on supply must be investigated at the industry level. We have constructed industry supply as the sum of contributions from individual deposits whose opening date and size are endogenous in the long run. Considering a price increase occurring at some strictly positive date (the beginning of the planning period being zero), we find that the date of opening of a deposit may only be unchanged or postponed by the future price increase. Comparing the substitution and the stock inventory effect at the aggregate level, we find that the former dominates the latter over the first part of the planning period but may fail to do so after the first new opening date of a deposit if any. However, when the cost of developing reserves of a new deposit is stationary, the substitution effect always dominates as in the case of supply from a single source. 


\section{References}

Adelman, M.A. (1990), "Mineral Depletion, with Special Reference to Petroleum", Review of Economics and Statistics, 72: 1-10.

Adelman, M.A. (1993), The Economics of Petroleum Supply, MIT Press.

Amigues, J.-P., P. Favard, G. Gaudet and M. Moreaux (1998), "On the Optimal Order of Natural Resource Use when the Capacity of the Inexhaustible Substitute is Limited", Journal of Economic Theory, 80: 153-170.

Amigues, J.-P., and M. Moreaux (2002), "On the Equilibrium Order of Exploitation of the Natural Resources", LERNA-TSE Working Papers 02.09.084.

Burness, H.S. (1976), "On the Taxation of Nonreplenishable Natural Resources", Journal of Environmental Economics and Management, 3: 289-311.

Cairns, R.D. (1990), "The Economics of Exploration for Non-renewable Resources", Journal of Economic Surveys, 4: 361-395.

Dasgupta, P.S., G.M. Heal and J.E. Stiglitz (1981), "The Taxation of Exhaustible Resources", NBER Working Papers 436.

Fischer, C., and R. Laxminarayan (2005), "Sequential Development and Exploitation of an Exhaustible Resource: Do Monopoly Rights Promote Conservation?", Journal of Environmental Economics and Management, 49: 500-515.

Fischer, C., and S.W. Salant (2013), "Limits to Limiting Greenhouse Gases: Intertemporal Leakage, Spatial Leakage, and Negative Leakage", mimeo, University of Michigan.

Gaudet, G., and P. Lasserre (1988), "On Comparing Monopoly and Competition in Exhaustible Resource Exploitation", Journal of Environmental Economics and Management, 15: 412-418.

Gaudet, G., and P. Lasserre (2011), "The Efficient Use of Multiple Sources of a Nonrenewable Resource under Supply Cost Uncertainty", International Economic Review, 52: $245-258$.

Gaudet, G., and P. Lasserre (2013), "The Taxation of Nonrenewable Natural Resources", in: Handbook on the Economics of Natural Resources, Eds. R. Halvorsen and D. F. Layton, Edward Elgar.

Gaudet, G., M. Moreaux and S.W. Salant (2001), "Intertemporal Depletion of Resource Sites by Spatially Distributed Users", American Economic Review, 91: 1149-1159.

Gerlagh, R. (2011), "Too Much Oil", CESifo Economic Studies, 57: 79-102. 
Golosov, M., J. Hassler, P. Krusell, and A. Tsyvinski (2014), "Optimal Taxes on Fossil Fuel in General Equilibrium", Econometrica, 82: 41-88.

Gordon, R.L. (1967), "A Reinterpretation of the Pure Theory of Exhaustion", Journal of Political Economy, 75: 274-286.

Grafton, R.Q., T. Kompas and N.V. Long (2012), "Substitution between Biofuels and Fossil Fuels: Is there a Green Paradox", Journal of Environmental Economics and Management, 64: 328-341.

Gray, L.C. (1914), "Rent Under the Assumption of Exhaustibility", Quarterly Journal of Economics, 28: 466-489.

Harstad, B. (2012), "Buy Coal! A Case for Supply-Side Environmental Policy", Journal of Political Economy, 120: 77-115.

Hartwick, J.M. (1978), "Exploitation of Many Deposits of an Exhaustible Resource" Econometrica, 46: 201-217.

Hartwick, J.M., M.C. Kemp, N.V. Long (1986), "Set-Up Costs and Theory of Exhaustible Resources", Journal of Environmental Economics and Management, 13: 212-24.

Herfindahl, O.C. (1967), "Depletion and Economic Theory", in: Extractive Resources and Taxation, Ed. M. Gaffney, University of Wisconsin Press: 63-90.

Hoel, M. (2012), "Carbon Taxes and the Green Paradox", in: Climate Change and Common Sense: Essays in Honour of Tom Schelling, Eds. R.W. Hahn and A. Ulph, Oxford University Press: 203-224.

Hotelling, H. (1931), "The Economics of Exhaustible Resources", Journal of Political Economy, 39: 137-175.

Kemp, M.C., and N.V. Long (1980) "On Two Folk Theorems Concerning the Extraction of Exhaustible Resources", Econometrica, 48: 663-673.

Livernois, J. R. and R. S. Uhler (1987), "Extraction Costs and the Economics of Nonrenewable Resources", Journal of Political Economy, 95: 195-203.

Levhari, D., and N. Liviatan (1977), "Notes on Hotelling's Economics of Exhaustible Resources", Canadian Journal of Economics, 10: 177-192.

Long, N.V., and H.-W. Sinn (1985), "Surprise Price Shifts, Tax Changes and the Supply Behaviour of Resource Extracting Firms", Australian Economic Papers, 24: 278-289.

McFadden, D.L. (1978), "Duality of Production, Cost, and Profit Functions", in: Production Economics: A Dual Approach to Theory and Applications, Vol. I: The Theory of Production, Eds. M.A. Fuss and D.L. McFadden, North-Holland: 2-109. 
Pindyck, R.S. (1978), "The Optimal Exploration and Production of Nonrenewable Resources", Journal of Political Economy, 86: 841-861.

van der Ploeg, F., and C. Withagen (2012a), "Is There Really a Green Paradox?", Journal of Environmental Economics and Management, 64: 342-363.

van der Ploeg, F., and C. Withagen (2012b), "Too Much Coal, Too Little Oil", Journal of Public Economics, 96: 62-77.

van der Ploeg, F., and C. Withagen (2014), "Growth, Renewables and the Optimal Carbon Tax", International Economic Review, 55: 283-311.

Quyen, N.V. (1988), "The Optimal Depletion and Exploration of a Nonrenewable Resource", Econometrica, 56: 1467-1471.

Salant, S.W., M. Eswaran and T.R. Lewis (1983), "The Length of Optimal Extraction Programs When Depletion Affects Extraction Costs", Journal of Economic Theory, 31: 364-374.

Salant, S.W. (2013), "The Equilibrium Price Path of Timber in the Absence of Replanting: Does Hotelling Rule the Forests Too?", Resource and Energy Economics, 35: $572-581$.

Sinn, H.-W. (2008), "Public Policies Against Global Warming: A Supply Side Approach", International Tax and Public Finance, 15: 360-394.

Slade, M.E. (1988), "Grade Selection under Uncertainty: Least Cost Last and Other Anomalies", Journal of Environmental Economics and Management, 15: 189-205.

Sweeney, J.L. (1993), "Economic Theory of Depletable Resources: An Introduction", in: Handbook of Natural Resource and Energy Economics, Vol. III, Eds. A.V. Kneese and J. L. Sweeney, Elsevier: 759-854.

Venables, A.J. (2014), "Depletion and Development: Natural Resource Supply with Endogenous Field Opening", Journal of the Association of Environmental and Resource Economists, 1: 313-336.

Weitzman, M.L. (1976), "The Optimal Development of Resource Pools", Journal of Economic Theory, 12: 351-364. 


\section{APPENDIX (FOR ONLINE PUBLICATION)}

\section{A Hotelling-Gordon Model: Stock Effects in Extraction Costs}

\section{1.a Problem Statement and Preliminaries}

The problem under investigation is (31)-(35). In this problem, (32) and (33) imply that $X_{t+1} \leq X_{t}$ for all $t \geq 0$. Thus it is sufficient that (35) be imposed when $t \rightarrow \infty$ or at the highest date considered. Let us call $X_{\infty}$ the value of $X_{t}$ at that date or its limit when $t \rightarrow \infty$. Then (35) can be replaced by

$$
X_{\infty} \geq X^{F} \geq 0
$$

We denote $\mu_{t}$ and $\eta_{t}$, the Lagrange multipliers respectively associated with date$t$ constraints (32) and (33); the Lagrange multipliers associated with $X_{\infty} \geq X^{F}$ and $X^{F} \geq 0$ are $\lambda$ and $\varepsilon$. The Lagrangian is

$$
\begin{aligned}
\mathcal{L} & =\sum_{t \geq 0}\left(p_{t} x_{t}-C_{t}\left(x_{t}, X_{t}\right)+\eta_{t} x_{t}\right)-E\left(X^{0}-X^{F}\right) \\
& +\sum_{t \geq 0} \mu_{t}\left(X_{t}-x_{t}-X_{t+1}\right)+\lambda\left(X_{\infty}-X^{F}\right)+\varepsilon X^{F} .
\end{aligned}
$$

It must be maximized with respect to $x_{t}$ and $X_{t}$ at all dates in both the long-run and the short-run versions of the problem. In the long-run version it is also maximized with respect to $X^{F}$, resulting in the long-run supply and cumulative supply functions $x_{t}^{*}(p)$ and $X_{t}^{*}(p)$. In the short-run version $X^{F}$, or equivalently developed reserves $X^{0}-X^{F}$, is taken as given, resulting in the short-run (or restricted) supply and cumulative supply functions $\widetilde{x}_{t}\left(p, X^{0}-X^{F}\right)$ and $\widetilde{X}_{t}\left(p, X^{0}-X^{F}\right)$.

$\mathcal{L}$ is strictly concave in $X^{F}$ by the convexity of $E ; \mathcal{L}$ is strictly concave in $x_{t}$ and $X_{t}$ by the convexity of $C_{t}$; the latter means that

$$
\frac{\partial^{2} C_{t}(x, X)}{\partial x^{2}}, \frac{\partial^{2} C_{t}(x, X)}{\partial X^{2}}, \text { and } \frac{\partial^{2} C_{t}(x, X)}{\partial x^{2}} \frac{\partial^{2} C_{t}(x, X)}{\partial X^{2}}-\left(\frac{\partial^{2} C_{t}(x, X)}{\partial x \partial X}\right)^{2},
$$

are all strictly positive for all $t \geq 0$, for all $X \geq 0$ and all $x>0$.

Differentiating (47) with respect to $x_{t}$ and $X_{t}$ gives the following first-order conditions:

$$
\mu_{t}-\eta_{t}=p_{t}-\frac{\partial C_{t}\left(x_{t}, X_{t}\right)}{\partial x}, \eta_{t} \geq 0, x_{t} \eta_{t}=0, \forall t \geq 0
$$

and

$$
\mu_{t-1}=\mu_{t}-\frac{\partial C_{t}\left(x_{t}, X_{t}\right)}{\partial X}, \forall t \geq 0 .
$$

Let $S$ be defined as the last date at which extraction is strictly positive; $S$ is endogenous; we assume for simplicity that $S \geq 1$. Also for simplicity, we assume that $x_{0}>0 .{ }^{36}$ Thus,

$$
x_{0}>0, x_{t} \geq 0, \forall t=1, \ldots, S-1, x_{S}>0 \text { and } x_{t}=0, \forall t>S,
$$

\footnotetext{
${ }^{36}$ The proofs of this appendix extend to the case where the deposit is not exploited at date 0 , or only exploited at date 0 . The results are also valid when $S \rightarrow \infty$.
} 
and, by (32),

$$
X_{t}=X_{S+1}, \forall t \geq S+1
$$

Since the development of reserves is costly, the optimum plans of the producer will bind the first exhaustibility constraint, $X_{\infty} \geq X^{F}$, in (46). Precisely, starting at $t=S+1$, $X_{t}=X_{t+1}$ by (52) so that $X_{t}$ must reach $X_{\infty}=X^{F}$ at $S+1$ if it is to equal $X^{F}$ at the end of the program. Consequently,

$$
\begin{aligned}
& X_{t}=X^{F}, x_{t}=0, \eta_{t} \geq 0, \forall t \geq S+1 \\
& X_{t}>X^{F}, x_{t} \geq 0, \eta_{t} \geq 0, \eta_{t} x_{t}=0, \forall t \leq S .
\end{aligned}
$$

It follows from (49) that

$$
\mu_{t}=p_{t}-\frac{\partial C_{t}\left(x_{t}, X_{t}\right)}{\partial x}, x_{t}>0, \forall t=0, \ldots, S
$$

which is expression (36) in the text. If $x_{t}=0$, either

$$
\mu_{t} \geq p_{t}-\frac{\partial C_{t}\left(0, X_{t}\right)}{\partial x}, \forall t \leq S
$$

or

$$
\mu_{t} \geq p_{t}-\frac{\partial C_{t}\left(0, X^{F}\right)}{\partial x}, \forall t \geq S+1
$$

Since $C_{t}(0, X)=0$ for all $X \geq 0, \frac{\partial C_{t}(0, X)}{\partial X}=0$ for all $X \geq 0$. Therefore, before $S,(50)$ implies

$$
\mu_{t-1}=\mu_{t}, \text { if } x_{t}=0, \forall t<S .
$$

Beyond $S$, since $x_{t}=0,(50)$ reduces to

$$
\mu_{t-1}=\mu_{t}, \forall t \geq S+1
$$

(50) is unchanged at $t \leq S$ when extraction is strictly positive:

$$
\mu_{t-1}=\mu_{t}-\frac{\partial C_{t}\left(x_{t}, X_{t}\right)}{\partial X}, x_{t}>0, \forall t=0, \ldots, S
$$

which is expression (37) in the text.

Since, starting at $t=S+1, x_{t}=0$ and $X_{t}=X_{t+1}$ by (51) and (52), the sum $\sum_{t \geq 0} \mu_{t}\left(X_{t}-x_{t}-X_{t+1}\right)$ in the Lagrangian (47) reduces to $\sum_{t=0, \ldots, S} \mu_{t}\left(X_{t}-x_{t}-X_{t+1}\right)$. Consequently, the Lagrangian may be rewritten

$$
\begin{aligned}
\mathcal{L} & =\sum_{t \geq 0}\left(p_{t} x_{t}-C_{t}\left(x_{t}, X_{t}\right)+\eta_{t} x_{t}\right)-E\left(X^{0}-X^{F}\right) \\
& +\sum_{t=0, \ldots, S} \mu_{t}\left(X_{t}-x_{t}-X_{t+1}\right)+\lambda\left(X_{\infty}-X^{F}\right)+\varepsilon X^{F}
\end{aligned}
$$


where (52) also implies that $X_{S+1}$ in the term $\mu_{S}\left(X_{S}-x_{S}-X_{S+1}\right)$ equals $X_{\infty}$. It follows that the first-order condition to the choice of $X_{\infty}$ requires the equality $\mu_{S}=\lambda$. Since $\mu_{t}$ remains constant starting at $t=S$ by (59), we obtain

$$
\mu_{t}=\lambda, \forall t \geq S
$$

This gives the equality on the left-hand side of (38) in the text.

When $X^{F}$ is treated as endogenous (long run) and the constraint $X^{F} \geq 0$ in (46) is binding, the first-order condition associated with $X^{F}$ in (47) is ${ }^{37}$

$$
E^{\prime}\left(X^{0}\right)=\lambda-\varepsilon, X^{F}=0, \lambda>0, \varepsilon \geq 0 ;
$$

when $X^{F}>0$, it must satisfy

$$
E^{\prime}\left(X^{0}-X^{F}\right)=\lambda, X^{F}>0, \lambda>0, \varepsilon=0 .
$$

This establishes the right-hand-side equality in (38).

Let us define the short-run value function

$$
\mathcal{V}\left(p, X^{0}-X^{F}\right) \equiv \sum_{t \geq 0}\left(p_{t} x_{t}-C_{t}\left(x_{t}, X_{t}\right)\right)
$$

where $x_{t}$ and $X_{t}$ are solutions $\widetilde{x}_{t}\left(p, X^{0}-X^{F}\right)$ and $\widetilde{X}_{t}\left(p, X^{0}-X^{F}\right)$ to the restricted $\left(X^{0}-X^{F}\right.$ fixed) version of Problem (31)-(35), i.e. satisfy (55), (56), (57), (58), (59) and (60). $\mathcal{V}\left(p, X^{0}-X^{F}\right)$ denotes the total present-value revenue derived from the exploitation of the developed reserves $X^{0}-X^{F}$. By standard interpretation, $\frac{\partial \mathcal{V}\left(p, X^{0}-X^{F}\right)}{\partial\left(X^{0}-X^{F}\right)}$ is the implicit value of the marginal extracted unit, which is also the implicit value $\lambda$ of the marginal developed reserve unit:

$$
\lambda \equiv \frac{\partial \mathcal{V}\left(p, X^{0}-X^{F}\right)}{\partial\left(X^{0}-X^{F}\right)}>0 .
$$

By the assumption of cost convexity, $\mathcal{V}$ is increasing and strictly concave in $X^{0}-X^{F}$.

In the long run, when $X^{F}$ is endogenous, so that (63) and (64) hold; it follows

$$
\begin{gathered}
E^{\prime}\left(X^{0}\right)=\frac{\partial \mathcal{V}\left(p, X^{0}\right)}{\partial\left(X^{0}\right)}-\varepsilon, X^{F}=0 ; \varepsilon \geq 0, \lambda>0 ; \\
E^{\prime}\left(X^{0}-X^{F}\right)=\frac{\partial \mathcal{V}\left(p, X^{0}-X^{F}\right)}{\partial\left(X^{0}-X^{F}\right)}, X^{F}>0, \varepsilon=0, \lambda>0 .
\end{gathered}
$$

When the constraint $X^{F} \geq 0$ on the availability of geological reserves is not binding, reserves are developed in such a way that the cost of developing the marginal unit is equal to the contribution of this unit to the intertemporal profit. However, in the case where the marginal cost of developing the totality of geological reserves falls short of the value of the marginal reserve unit, it is optimal to set $X^{F}=0$, with $0<E^{\prime}\left(X^{0}\right)=\lambda-\varepsilon<\lambda$.

In the short run, when $X^{0}-X^{F}$ is given, (66) holds, but conditions (63) and (64) are not necessarily satisfied.

\footnotetext{
${ }^{37}$ Given our assumptions on prices and costs, a strictly positive stock of reserves $X^{0}-X^{F}$ is developed and exploited. Thus $E^{\prime}\left(X^{0}-X^{F}\right)>0$, which will imply that $\lambda>0$.
} 


\section{1.b Proof of Proposition 4}

\section{Stock effect on restricted cumulative supply}

Assuming that exploitable reserves $X^{0}-X^{F}$ are parametric, with $X_{S+1}=X^{F}$ as per (53), consider an increase $d\left(X^{0}-X^{F}\right)>0$, i.e. a reduction $d X^{F}<0$ ( $X^{0}$ is given); this requires $X^{F}>0$. In the sequel, we establish the effect on the restricted supply and restricted cumulative supply functions $\widetilde{x}_{t}\left(p, X^{0}-X^{F}\right)$ and $\widetilde{X}_{t}\left(p, X^{0}-X^{F}\right)$ of this exogenous reserve increase; $\widetilde{x}_{t}\left(p, X^{0}-X^{F}\right)$ and $\widetilde{X}_{t}\left(p, X^{0}-X^{F}\right)$ are the values of $X_{t}$ and $x_{t}$ in the solution of Problem (31)-(35), whose Lagrangian is (47), when $x_{t}$ and $X_{t}$ are endogenous but $X^{F}$ is exogenous.

The strict concavity of $\mathcal{V}$ in (66) implies that the rise in reserves causes a strict reduction in $\lambda$. The final extraction date $S$ may be modified as a result of the reserve change. In what follows, we will denote by $S$ the date at which extraction endogenously stops once the reserve change is taken into account.

Notation 1 If $S$ is modified by any parametric change, $S$ denotes in these proofs the date at which extraction endogenously stops once the parameter change is taken into account. For example, if $S$ changes from $S_{0}$ to $S_{1}$ as a result of a change in $X^{F}$ from $X_{0}^{F}$ to $X_{1}^{F}$, the notation $x_{S}$ signifies $x_{S_{1}}$ and $d x_{S}$ signifies $\left.x_{S_{1}}\right|_{X^{F}=X_{1}^{F}}-\left.x_{S_{1}}\right|_{X^{F}=X_{0}^{F}}$.

The proof makes use of several lemmas.

Lemma 2 As a result of a reserve change $d\left(X^{0}-X^{F}\right)>0, d \lambda<0, d \mu_{S}<0$ and $d X_{S+1}<0$.

$d \lambda<0$ is shown above.

If $S$ is unchanged, $\mu_{S}=\lambda$ and $X_{S+1}=X^{F}$ before and after the reserves' change; the lemma immediately holds in that case.

If $S$ is postponed, for all $t \geq S, \mu_{t}=\mu_{S}=\lambda$ by (62) and $X_{t+1}=X_{S+1}=X^{F}$, before and after the change; the lemma also immediately holds.

Examine now the case where $S$ is advanced. Before the change, $\mu_{t}>\lambda$ by (60) and $X_{t+1}>X^{F}$ for all $t$ strictly preceding the initial last-extraction date; in particular at the date $S$ which will be the last extraction date after the change, remembering Notation 1, $X_{S+1}>X^{F}$. After the change, since $\mu_{S}=\lambda$ and $X_{S+1}=X^{F}$ while the change implies $d \lambda<0$ and $d X^{F}<0$, it follows that $d \mu_{S}<0$ and $d X_{S+1}<0$.

The following lemma will be used later to exploit Lemma 2 .

Lemma $3 \mathrm{If}$, as a result of the increase in reserves, $d \mu_{t} \geq 0$ and $d X_{t+1} \geq 0$ for some $t=0, \ldots, S-1$, then $d \mu_{t+1} \geq 0$ and $d X_{t+2} \geq 0$.

For some date $t=0, \ldots, S-1$, assume that the change in reserves causes changes $d \mu_{t} \geq 0$ and $d X_{t+1} \geq 0$.

If $x_{t+1}$ was null and remains so as a result of the change in reserves, then $\mu_{t+1}=\mu_{t}$ by (58); it follows that $d \mu_{t+1} \geq 0$. Also, $d x_{t+1}=0$ implies by (32) that $d X_{t+2}=d X_{t+1} \geq 0$.

Consider now that $x_{t+1}$ was strictly positive and remains so after the change, so that (55) holds. Assume, as a premise to be contradicted, that $d \mu_{t+1}<0$. On the one hand, 
total differentiation of (55) at date $t+1$ then implies

$$
d x_{t+1}>\frac{-\frac{\partial^{2} C_{t+1}}{\partial x \partial X}}{\frac{\partial^{2} C_{t+1}}{\partial x^{2}}} d X_{t+1}
$$

On the other hand, totally differentiating (60) and (55), taken at $t+1$, and substituting yield

$$
d \mu_{t}=-\left[\frac{\partial^{2} C_{t+1}}{\partial x^{2}}+\frac{\partial^{2} C_{t+1}}{\partial x \partial X}\right] d x_{t+1}-\left[\frac{\partial^{2} C_{t+1}}{\partial X \partial x}+\frac{\partial^{2} C_{t+1}}{\partial X^{2}}\right] d X_{t+1}
$$

The first term between brackets is strictly positive by assumption (30). Using (67), and simplifying it follows that

$$
d \mu_{t}<\left(\frac{\partial^{2} C_{t+1}}{\partial x^{2}}\right)^{-1}\left[\left(\frac{\partial^{2} C_{t+1}}{\partial x \partial X}\right)^{2}-\frac{\partial^{2} C_{t+1}}{\partial x^{2}} \frac{\partial^{2} C_{t+1}}{\partial X^{2}}\right] d X_{t+1}
$$

where assumption (48) implies that the term multiplying $d X_{t+1}$ is negative. Thus the lemma's assumption $d X_{t+1} \geq 0$ implies that $d \mu_{t}<0$. This contradicts the other lemma's assumption $d \mu_{t} \geq 0$. We conclude that the maintained assumption $d \mu_{t+1}<0$ cannot hold and that $d \mu_{t+1} \geq 0$ when $x_{t+1}$ remains positive as a result of the change.

Consider finally the (intermediate) cases where extraction is zero before the reserve change and becomes strictly positive as a result of the change, or vice versa, where extraction is strictly positive and becomes zero. We just showed that $d \mu_{t+1} \geq 0$ if extraction is null and remains so, and also if extraction is strictly positive and remains so. By the Maximum Theorem $\mu_{t+1}$ is continuous across these cases, so that we also have $d \mu_{t+1} \geq 0 .{ }^{38}$

We have shown that $d \mu_{t+1} \geq 0$; now consider $d X_{t+2}$. Consider first that extraction $x_{t+2}$ was null before the reserve change and remains so after the change. In that case, (32) implies that $d X_{t+2}=d X_{t+1}$, which is positive as lemma assumption. Consider now that extraction $x_{t+2}$ was and remains strictly positive. In that case, (55) holds before and after the reserve change, so that the rise $d \mu_{t+1} \geq 0$ implies a reduction in $\frac{\partial C_{t+1}}{\partial x}$, that is by total differentiation of (55)

$$
d x_{t+1} \leq \frac{-\frac{\partial^{2} C_{t+1}}{\partial x \partial X}}{\frac{\partial^{2} C_{t+1}}{\partial x^{2}}} d X_{t+1}
$$

\footnotetext{
${ }^{38}$ The Maximum Theorem applies as follows to the restricted problem under study. The exploitable reserves parameter $X^{0}-X^{F}$ continuously affects the extraction possibility set defined by (32), (33) and (35), and continuously affects the objective (31). Since $X^{0}-X^{F}$ must be finite by (35) and by the finiteness of $X^{0}$, the extraction possibility set is bounded, and is evidently closed. Furthermore, the objective (31) is strictly concave by assumption and for any given reserves level $X^{0}-X^{F}$, the set of extraction possibilities is convex since the convex combination of two possible extraction paths satisfying the exhaustibility constraint satisfies the exhaustibility constraint. The Maximum Theorem thus applies, implying that the optimum extraction path $\left(x_{t}, X_{t}\right)_{t \geq 0}$ is a continuous function of the reserve level $X^{0}-X^{F}$. In turn, because all multipliers $\mu_{t}, t \geq 0$, are defined as continuous functions of $x_{t}$ and $X_{t}$ by (55) and (59), it follows that in optimum they are continuous functions of $X^{0}-X^{F}$.
} 
By assumptions (29) and (30), the coefficient of $d X_{t+1}$ in the above inequality is positive and lower than unity. Thus the inequality implies that $d x_{t+1} \leq d X_{t+1}$, which yields $d X_{t+2} \geq 0$. The continuity argument made earlier to invoke the Maximum Theorem also applies here for cases where extraction $x_{t+2}$ becomes positive or null as a result of the change in reserves.

The combination of Lemma 2 and Lemma 3 will give the following result.

Lemma 4 As a result of the reserve increase, $d \mu_{0}<0$ and $d X_{1}<0$, and for all $t=$ $1, \ldots, S$, either $d \mu_{t}<0$ or $d X_{t+1}<0$.

This lemma follows from Lemma 2 and the contrapositive of the series of implications in Lemma 3. Indeed, the final implication of Lemma 3 that $\mu_{S} \geq 0$ is contradicted by Lemma 2. Thus for all $t=0, \ldots, S, d \mu_{t} \geq 0$ and $d X_{t+1} \geq 0$ do not hold at the same time: either $d \mu_{t}<0$ or $d X_{t+1}<0$, where the relation "or" is not exclusive.

For $t=0, x_{0}>0$ by (51) so that (55) holds. It follows that $d \mu_{0}<0$ is equivalent to $d x_{0}>0$ since $X^{0}$ is given, which is equivalent by (32) to $d X_{1}<0$. Thus the proposition that "either $d \mu_{0}<0$ or $d X_{1}<0$ holds" is equivalent to " $d \mu_{0}<0$ and $d X_{1}<0$ hold".

The result that $d \mu_{0}<0$ and $d X_{1}<0$ will be later exploited by use of the following lemma, whose proof partly relies on the other part of Lemma 4.

Lemma 5 If, as a result of the reserve increase, $d \mu_{t}<0$ and $d X_{t+1}<0$ for some $t=0, \ldots, S-1$, then $d \mu_{t+1}<0$ and $d X_{t+2}<0$.

For some $t=0, \ldots, S-1$, assume that $d \mu_{t}<0$ and $d X_{t+1}<0$ hold simultaneously.

Suppose, as an assumption to be contradicted, that $d X_{t+2} \geq 0$.

There are several possibilities as far as extraction $x_{t+1}$ is affected by the change in reserves. First consider the case where $x_{t+1}=0$ before and after the change. Then by (32), $d X_{t+2}=d X_{t+1}<0$, which contradicts the maintained assumption.

Consider now the case where extraction $x_{t+1}$ was strictly positive before the change in reserves and remains so after the change. By Lemma $4, d X_{t+2} \geq 0$ implies

$$
d \mu_{t+1}<0
$$

On the other hand, by (32), $d X_{t+2} \geq 0$ implies

$$
d X_{t+1} \geq d x_{t+1}
$$

Differentiating (55) at $t+1$ gives

$$
d \mu_{t+1}=-\frac{\partial^{2} C_{t+1}}{\partial x^{2}} d x_{t+1}-\frac{\partial^{2} C_{t+1}}{\partial x \partial X} d X_{t+1},
$$

where the coefficient of $d x_{t+1}$ is strictly negative by assumption (48). Substituting for $d x_{t+1}$ by use of inequality (71) implies

$$
d \mu_{t+1} \geq-\left[\frac{\partial^{2} C_{t+1}}{\partial x^{2}}+\frac{\partial^{2} C_{t+1}}{\partial x \partial X}\right] d X_{t+1},
$$


where the term between brackets is strictly positive by (30). Since $d X_{t+1}<0$ by assumption of the lemma, the inequality implies that $d \mu_{t+1}>0$, which contradicts $(70)$.

Therefore, whether extraction at $t+1$ remains zero or remains strictly positive as a result of the reserve change, it must be true that $d X_{t+2}<0$. Finally consider the (intermediate) cases where $x_{t+1}$ was zero and becomes strictly positive or, vice versa, was strictly positive and becomes zero. By the Maximum Theorem (see Footnote 38 on how it applies here), for any $t \geq 0, X_{t+2}$ is continuous in reserves. Hence, the result that $d X_{t+2}<0$ also applies across the cases where extraction remains zero or strictly positive, that is in the intermediate cases.

We conclude that, whether extraction was and remains zero or becomes strictly positive, or else was strictly positive and remains so or falls to zero at date $t+1$,

$$
d X_{t+2}<0
$$

It follows by (32) that

$$
d X_{t+1}<d x_{t+1}
$$

Let us now show that $d \mu_{t+1}<0$. Consider first that extraction $x_{t+1}$ was null before the reserve change and remains so after the change. In that case, $\mu_{t+1}=\mu_{t}$ before and after the change by (58) or (59). It follows that $d \mu_{t+1}=d \mu_{t}<0$ by the lemma's assumption that $d \mu_{t}<0$. Consider now that extraction $x_{t+1}$ was and remains strictly positive. In that case, (55) holds at $t+1$ before and after the reserve change, which gives $d \mu_{t+1}=-\frac{\partial^{2} C_{t+1}}{\partial x^{2}} d x_{t+1}-\frac{\partial^{2} C_{t+1}}{\partial x \partial X} d X_{t+1}$, where the coefficient of $d x_{t+1}$ is strictly negative by (48). Substituting for $d x_{t+1}$ by use of inequality (72) implies

$$
d \mu_{t+1} \leq-\left[\frac{\partial^{2} C_{t+1}}{\partial x^{2}}+\frac{\partial^{2} C_{t+1}}{\partial x \partial X}\right] d X_{t+1}
$$

where the term between brackets is strictly positive by (30). Since $d X_{t+1}<0$ by assumption of the lemma, the inequality implies that $d \mu_{t+1}<0$. The continuity argument invoked earlier (Maximum Theorem; see Footnote 38) also applies here for cases where extraction $x_{t+1}$ becomes positive or null as a result of the change in reserves.

The following lemma concludes the proof of Proposition 4.1.

Lemma 6 As a result of the increase in reserves, $d \mu_{t}<0$ and $d X_{t+1}<0$ for all $t=$ $0, \ldots, S$.

By Lemma $4, d \mu_{0}<0$. Since $x_{0}>0$ by assumption (51), (55) holds, where $X_{0}$ is fixed. It follows from $d \mu_{0}<0$ in (55) that $d x_{0}>0$. In turn, (32) implies $d X_{1}<0$.

$d \mu_{t}<0$ and $d X_{t+1}<0$ are thus simultaneously verified for $t=0$, which implies by Lemma 5 that $d \mu_{t+1}<0$ and $d X_{t+2}<0$, for all $t=0, \ldots, S-1$. Lemma 6 is obtained by recurrence, thus completing the proof of Proposition 4.1.

\section{Cross-price effects on restricted and unrestricted supply}

The following proof will simultaneously establish the effects of a price change on restricted cumulative supply $X^{0}-\widetilde{X}_{t}\left(p, X^{0}-X^{F}\right)$ and on unrestricted cumulative supply $X^{0}-X_{t}^{*}(p) . \quad X_{t}^{*}(p)$ is the value of $X_{t}$ in the solution of Problem (31)-(35), whose 
Lagrangian is (47), when $x_{t}, X_{t}$ and $X^{F}$ are treated as endogenous; $\widetilde{X}_{t}\left(p, X^{0}-X^{F}\right)$ is the value of $X_{t}$ in the solution of Problem (31)-(35) when $x_{t}$ and $X_{t}$ are endogenous but $X^{F}$ is exogenous.

We will consider a strict price rise $d p_{T}>0$ at some date $T \geq 1$ that differs from the pre-change date of last strictly-positive extraction, and is such that $x_{T}$ is strictly positive before the change; the proof can easily be extended to $T=0$ and to a price rise occurring at the pre-change date of last extraction. The restriction that $x_{T}>0$ rules out the uninteresting possibility that the price rise has no effect on $\lambda$ because it applies to a null extraction base. It also implies that the price rise does not occur at a date posterior to the pre-change date of last extraction.

When the size of reserves is restricted, according to (66), the value of the marginal unit of reserves is $\lambda=\frac{\partial \mathcal{V}\left(p, X^{0}-X^{F}\right)}{\partial\left(X^{0}-X^{F}\right)}$, with $\frac{\partial^{2} \mathcal{V}\left(p, X^{0}-X^{F}\right)}{\partial\left(X^{0}-X^{F}\right) \partial p_{T}}>0$. Thus the price rise $d p_{T}>0$ at some date of the extraction phase implies $d \lambda>0$.

When reserves are not restricted, there are two possibilities. When $X^{F}>0$, (64) and (66) hold: developed and exploited reserves $X^{0}-X^{F}$ and their marginal value $\lambda$ are jointly determined by the equality of the strictly rising marginal development cost function $E^{\prime}\left(X^{0}-X^{F}\right)$ with the decreasing marginal value of reserves $\frac{\partial \mathcal{V}\left(p, X^{0}-X^{F}\right)}{\partial\left(X^{0}-X^{F}\right)}$. In that context, a price rise $d p_{T}>0$ at some date of the extraction phase causes a rise $d \lambda>0$ and an increase in developed and exploited reserves $d\left(X^{0}-X^{F}\right)>0$ or, equivalently, $d X^{F}<0$. When $X^{F}=0$ before the price rise, the above effect does not take place: $d\left(X^{0}-X^{F}\right)=0$ despite the absence of reserve restriction so that $d \lambda>0$ as when reserves are restricted.

The following lemma gathers those results.

Lemma $7 \mathrm{~A}$ price rise $d p_{T}>0$ at some date $T \geq 1$ such that $x_{T}>0$ and $T$ strictly precedes the last date of strictly positive extraction, causes the value of the marginal reserve unit to increase strictly $(d \lambda>0)$ and developed reserves to increase $\left(d X^{F} \leq 0\right)$.

The possibility that $d X^{F}=0$ ensures that Lemma 7 holds whether supply is restricted or unrestricted and, in the latter case, whether the constraint $X^{F} \geq 0$ is binding or not.

The second step of this proof involves the backward recurrence described in Lemma 8. This recurrence will be used to assess the effect of the price change on quantities at the terminal date $S$ and then at all dates between $T$ and $S$.

Lemma 8 If, as a result of the date-T price rise, $d \mu_{t}>0$ and $d X_{t} \leq d x_{t} \leq 0$ for some date $t \leq S, t \neq T$, then $d \mu_{t-1}>0$ and $d X_{t-1} \leq d x_{t-1} \leq 0, t-1 \neq T$.

Assume that for some date $t \neq T, 1<t \leq S, d \mu_{t}>0$ and $d X_{t} \leq d x_{t} \leq 0$.

First, consider the case where $x_{t}$ was null before the price change and remains so afterwards. Then, $d x_{t}=0$, so that $\mu_{t-1}=\mu_{t}$ by (58). By the maintained assumption $d \mu_{t}>0$, it follows that $d \mu_{t-1}=d \mu_{t}>0$ in that case.

Second, consider the situation where $x_{t}$ was strictly positive before the change in price, and remains so with the change. (55) holds in that case; totally differentiating (55) 
at date $t$ and using $d \mu_{t}>0$ yields

$$
d x_{t}<\frac{-\frac{\partial^{2} C_{t}}{\partial x \partial X}}{\frac{\partial^{2} C_{t}}{\partial x^{2}}} d X_{t}
$$

Assume now that $t-1 \neq T$. Replacing $\mu_{t}$ in (60) by its expression as per (55) and totally differentiating give

$$
d \mu_{t-1}=-\left[\frac{\partial^{2} C_{t}}{\partial x^{2}}+\frac{\partial^{2} C_{t}}{\partial x \partial X}\right] d x_{t}-\left[\frac{\partial^{2} C_{t}}{\partial X^{2}}+\frac{\partial^{2} C_{t}}{\partial X \partial x}\right] d X_{t}
$$

where the first term between brackets is strictly positive by assumption (30). Substituting $d x_{t}$ by use of inequality (73) and rearranging, we obtain

$$
d \mu_{t-1}>\left(\frac{\partial^{2} C_{t}}{\partial x^{2}}\right)^{-1}\left[\left(\frac{\partial^{2} C_{t}}{\partial x \partial X}\right)^{2}-\frac{\partial^{2} C_{t}}{\partial x^{2}} \frac{\partial^{2} C_{t}}{\partial X^{2}}\right] d X_{t}
$$

where by assumption (48) the term between parentheses is positive and the term between brackets is negative. Since $d X_{t} \leq 0$ by the assumption of this proof, it follows from (74) that $d \mu_{t-1}>0$ also in that case.

One can conclude that the rise in price yields $d \mu_{t-1}>0$, whether $x_{t}$ decreases and remains strictly positive or $x_{t}$ is and remains zero. The Maximum Theorem implies that $\mu_{t-1}$ is continuous ${ }^{39}$ across these cases, so that $d \mu_{t-1}>0$ also holds as a result of the price rise when $x_{t}$ decreases from a strictly positive level to zero.

As far as date $t-1$ is concerned, there are two possibilities. If $x_{t-1}$ was and remains zero following the price rise, $d x_{t-1}=0$ so that (32) implies $d X_{t-1}=d X_{t} \leq 0$ by the maintained assumptions. Therefore, $d X_{t-1} \leq 0=d x_{t-1}$; the lemma applies in that case.

When $x_{t-1}>0$, the maintained assumption $d X_{t} \leq 0$ implies by (32) that

$$
d X_{t-1} \leq d x_{t-1}
$$

On the other hand, the differentiation of (55) at $t-1$ gives $d \mu_{t-1}=-\frac{\partial^{2} C_{t-1}}{\partial x^{2}} d x_{t-1}-$ $\frac{\partial^{2} C_{t-1}}{\partial x \partial X} d X_{t-1}$, where $\frac{\partial^{2} C_{t-1}}{\partial x \partial X}$ is strictly negative by assumption (29). Substituting for $d X_{t-1}$

${ }^{39}$ The Maximum Theorem applies to price changes in the context of this proof as explained shortly below. The continuity of multipliers $\mu_{t}$, for all dates $t \geq 0$ follows because those multipliers are continuous functions of $x_{t}$ and $X_{t}$ variables and price parameters by (55), (58) and (59). Whether Problem (31)(35) is restricted $\left(X^{0}-X^{F}\right.$ given) or not $\left(X^{F}\right.$ free), the Maximum Theorem applies as follows, when the parameters of interest are prices. The extraction possibility set defined by (32), (33) and (35), is independent of price parameters, hence continuous. Price parameters also continuously affect the objective (31). Even in the unrestricted problem, the finiteness of geological reserves $X^{0}$ ensures that developed reserves $X^{0}-X^{F} \geq 0$ are finite. Thus the extraction possibility set is bounded, and is evidently closed. Furthermore, the objective (31) is strictly concave by assumption and for any vector of prices, the set of extraction possibilities is convex since the convex combination of two possible extraction paths satisfying the exhaustibility constraint satisfies the exhaustibility constraint. The Maximum Theorem thus applies, implying that the optimum extraction path $\left(x_{t}, X_{t}\right)_{t \geq 0}$ is a continuous function of any component of the price vector. 
by use of inequality (75) thus yields

$$
d \mu_{t-1}<-\left[\frac{\partial^{2} C_{t-1}}{\partial x^{2}}+\frac{\partial^{2} C_{t-1}}{\partial x \partial X}\right] d x_{t-1}
$$

where the term between brackets is positive by assumption (30). We have shown above that $d \mu_{t-1}>0$, so that $d x_{t-1}$ must be strictly negative. (75) thus implies $d X_{t-1} \leq$ $d x_{t-1}<0$.

In the case where $x_{t-1}$ decreases with the price rise in such a way that it becomes zero, the latter inequality must be adjusted to $d X_{t-1} \leq d x_{t-1} \leq 0$; the lemma also applies.

Let us now examine the effect of the price change at the last extraction date $S$.

Lemma 9 Following the date-T price rise, at the date $S$ of last strictly positive extraction, $d \mu_{S}>0$ and $d X_{S} \leq d x_{S}<0$.

The date of last extraction may change as a result of the price rise considered in Lemma 7. However it cannot be postponed. Date $T$ of the price rise does not occur at a date with no extraction; thus $T$ is not posterior to the pre-change last extraction date. Therefore if $S$ is a date at which extraction had already stopped before the price rise, condition (57) had to hold before the price rise; considering (62), this implies that

$\mu_{t}=\lambda>p_{t}-\frac{\partial C_{t}\left(0, X^{F}\right)}{\partial x}$, where by Lemma 7 the price change induces $d \lambda>0$ and $d X^{F} \leq 0$. It follows from (29) that $d X^{F} \leq 0$ does not reduce the marginal cost $\frac{\partial C_{t}\left(0, X^{F}\right)}{\partial x}$; as a result, no rise $d x_{t}>0$ can cause equality (55) to be satisfied. However, following the price rise, (55) must hold at $S$ by definition of the after-change last strictly-positive extraction date.

Thus $S$ can only be advanced or left unchanged by the price rise. Then the equalities $\mu_{S}=\lambda$ and $X_{S+1}=X^{F}$ hold as per (62) and (53) respectively. Thus Lemma 7 implies $d \mu_{S}>0$ and $d X_{S+1} \leq 0$; the latter is equivalent by (32) to $d X_{S} \leq d x_{S}$. Totally differentiating (55) and substituting $d X_{S}$ by use of the latter inequality yield

$$
d \mu_{S} \leq-\left[\frac{\partial^{2} C_{S}}{\partial x^{2}}+\frac{\partial^{2} C_{S}}{\partial x \partial X}\right] d x_{S}
$$

where the term between brackets is positive by assumption (30). Since $d \mu_{S}>0$ in this case, it follows from inequality (76) that $d x_{S}<0$, which remains compatible with $x_{S}>0$. Therefore, $d X_{S} \leq d x_{S}<0$.

Lemma 10 immediately follows from the combination of Lemma 9 with Lemma 8's recurrence.

Lemma 10 As a result of the date-T price rise, $d \mu_{t}>0$ and $d X_{t} \leq d x_{t} \leq 0$, for all $t=T+1, \ldots, S$.

Let us now examine the effects of the price rise at the date $T$ when it occurs. We will establish the following lemma.

Lemma 11 As a result of the date-T price rise, $d x_{T}>0, d X_{T} \leq d x_{T}$, and $d \mu_{T}>0$. 
By Lemma 10,

$$
d X_{T+1} \leq d x_{T+1} \leq 0
$$

where $d X_{T+1} \leq 0$ implies

$$
d X_{T} \leq d x_{T}
$$

The law of supply prevails at date $T$, which implies, under Lemma 7's assumption that $x_{T}>0$, the strict inequality

$$
d x_{T}>0 .
$$

From Lemma 10, $d \mu_{T+1}>0$. Let us now show that $d \mu_{T}>0$.

Consider first the case $x_{T+1}=0$ before and after the price change; by (58), $\mu_{T}=\mu_{T+1}$. It follows that $d \mu_{T}=d \mu_{T+1}>0$.

Now consider the case where $x_{T+1}$ was strictly positive before the price change and remains so. Then, (55) holds; $d \mu_{T+1}>0$ implies

$$
d x_{T+1}<\frac{-\frac{\partial^{2} C_{T+1}}{\partial x \partial X}}{\frac{\partial^{2} C_{T+1}}{\partial x^{2}}} d X_{T+1}
$$

Taking (60) at $t=T+1$ and using (55) to eliminate $\mu_{T+1}$ gives

$$
d \mu_{T}=-\left[\frac{\partial^{2} C_{T+1}}{\partial x^{2}}+\frac{\partial^{2} C_{T+1}}{\partial x \partial X}\right] d x_{T+1}-\left[\frac{\partial^{2} C_{T+1}}{\partial X^{2}}+\frac{\partial^{2} C_{T+1}}{\partial X \partial x}\right] d X_{T+1},
$$

where the first term between brackets is strictly positive by assumption (30). Substituting $d x_{T+1}$ by use of inequality (79) and rearranging, one obtains

$$
d \mu_{T}>\left(\frac{\partial^{2} C_{T+1}}{\partial x^{2}}\right)^{-1}\left[\left(\frac{\partial^{2} C_{T+1}}{\partial x \partial X}\right)^{2}-\frac{\partial^{2} C_{T+1}}{\partial x^{2}} \frac{\partial^{2} C_{T+1}}{\partial X^{2}}\right] d X_{T+1}
$$

where by assumption (48) the term between parentheses is strictly positive and the term between brackets is strictly negative. Since $d X_{T+1} \leq 0$, the latter inequality implies that $d \mu_{T}>0$.

Finally consider the situation where $x_{T+1}$ was strictly positive before the change and decreases so as to become null with the price change. By the Maximum Theorem (see Footnote 39 ), the continuity of $x_{T+1}$ with the price guarantees that the above results apply in that situation.

The analysis will now turn to the effect of the price change on quantities at dates that precede the date $T$ of the change. We will first establish the following recurrence, that will be used shortly below.

Lemma 12 If, as a result of the price rise, $d \mu_{t} \leq 0$ and $d X_{t+1} \leq 0$ for some $t=$ $0, \ldots, T-2$, then $d \mu_{t+1} \leq 0$ and $d X_{t+2} \leq 0$.

For some $t=0, \ldots, T-2$, assume that $d \mu_{t} \leq 0$ and $d X_{t+1} \leq 0$ as a result of the price rise. 
Consider first the case where $x_{t+1}$ was zero before the price rise and remains so afterwards. In that case, by (58), $\mu_{t}=\mu_{t+1}$. It follows that $d \mu_{t+1}=d \mu_{t} \leq 0$. Moreover, differentiating (32) gives $d X_{t+2}=d X_{t+1}-d x_{t+1}$. With $d x_{t+1}=0$ in that case and $d X_{t+1} \leq 0$ by the maintained assumption, it follows $d X_{t+2} \leq 0$.

Second, consider the case where extraction $x_{t+1}$ initially was and remains strictly positive with the price rise so that (55) holds. Taking (60) at $t+1$, substituting for $d \mu_{t+1}$ using (55), and differentiating, we obtain

$$
d \mu_{t}=-\left[\frac{\partial^{2} C_{t+1}}{\partial x^{2}}+\frac{\partial^{2} C_{t+1}}{\partial x \partial X}\right] d x_{t+1}-\left[\frac{\partial^{2} C_{t+1}}{\partial X^{2}}+\frac{\partial^{2} C_{t+1}}{\partial X \partial x}\right] d X_{t+1}
$$

where the first term between brackets is strictly positive by assumption (30). Thus $d \mu_{t} \leq 0$ implies

$$
d x_{t+1} \geq \frac{-\left[\frac{\partial^{2} C_{t+1}}{\partial X^{2}}+\frac{\partial^{2} C_{t+1}}{\partial X \partial x}\right]}{\left[\frac{\partial^{2} C_{t+1}}{\partial x^{2}}+\frac{\partial^{2} C_{t+1}}{\partial x \partial X}\right]} d X_{t+1} .
$$

On the other hand, the differentiation of (55) at $t+1$ yields

$$
d \mu_{t+1}=-\frac{\partial^{2} C_{t+1}}{\partial x^{2}} d x_{t+1}-\frac{\partial^{2} C_{t+1}}{\partial x \partial X} d X_{t+1}
$$

where $-\frac{\partial^{2} C_{t+1}}{\partial x^{2}}$ is strictly negative by (48). Substituting for $d x_{t+1}$ using (80) and rearranging, one obtains

$$
d \mu_{t+1} \leq \frac{\left[\frac{\partial^{2} C_{t+1}}{\partial x^{2}} \frac{\partial^{2} C_{t+1}}{\partial X^{2}}-\left(\frac{\partial^{2} C_{t+1}}{\partial x \partial X}\right)^{2}\right]}{\left[\frac{\partial^{2} C_{t+1}}{\partial x^{2}}+\frac{\partial^{2} C_{t+1}}{\partial x \partial X}\right]} d X_{t+1},
$$

where the coefficient of $d X_{t+1}$ is strictly positive by (30) and (48). Thus the assumption $d X_{t+1} \leq 0$ implies $d \mu_{t+1} \leq 0$

With $d \mu_{t+1} \leq 0$, (81) implies

$$
d x_{t+1} \geq \frac{-\frac{\partial^{2} C_{t+1}}{\partial x \partial X}}{\frac{\partial^{2} C_{t+1}}{\partial x^{2}}} d X_{t+1}
$$

where the coefficient of $d X_{t+1}$ is positive and lower than unity by (30), while $d X_{t+1} \leq 0$ by the maintained assumption. It follows that $d x_{t+1} \geq d X_{t+1}$, which by (32) implies $d X_{t+2} \leq 0$.

Last, by continuity (see Footnote 39 on the Maximum Theorem), the above results also hold across cases, when $x_{t+1}$ becomes strictly positive or becomes zero following the price rise.

We can now show the following result.

Lemma 13 As a result of the price rise, $d \mu_{0}>0$ and, for all $t=1, \ldots, T$, either $d \mu_{t}>0$ or $d X_{t+1}>0$. 
As a result of Lemma 12's recurrence, if $d \mu_{t} \leq 0$ and $d X_{t+1} \leq 0$ for some $t \leq T-2$, then $d \mu_{T-1} \leq 0$ and $d X_{T} \leq 0$. Suppose, as an assumption to be contradicted, that $d \mu_{T-1} \leq 0$ and $d X_{T} \leq 0$. Differentiating (60) at $t=T$, we have

$$
d \mu_{T-1}=d \mu_{T}-\frac{\partial^{2} C_{T}}{\partial X \partial x} d x_{T}-\frac{\partial^{2} C_{T}}{\partial X^{2}} d X_{T}
$$

On the left-hand side, $d \mu_{T-1} \leq 0$ by assumption. On the right-hand side, the last term is positive by (48) and by the maintained assumption $d X_{T} \leq 0 ; d \mu_{T}>0$ by Lemma 11; and the second term is strictly positive by Lemma 11 and (29). Thus the right-hand side is strictly positive while the left-hand side in non positive. This contradiction implies that $d \mu_{T-1} \leq 0$ and $d X_{T} \leq 0$ do not hold simultaneously so that either $d \mu_{T-1}>0$ or (non exclusive) $d X_{T}>0$.

Now use the following contrapositive of Lemma 12's implication: if as a result of the price rise, for some $t=0, \ldots, T-2, d \mu_{t+1}>0$ or (non exclusive) $d X_{t+2}>0$, then either $d \mu_{t}>0$ or (non exclusive) $d X_{t+1}>0$. By backward recurrence, starting from the result established above that either $d \mu_{T-1}>0$ or (non exclusive) $d X_{T}>0$, it follows that, for all $t=0, \ldots, T-1$, either $d \mu_{t}>0$ or (non exclusive) $d X_{t+1}>0$. At date $t=0$, we have already shown by differentiation of (55) where $X_{0}$ is given, that $d \mu_{0}>0$ is equivalent to $d x_{0}<0$, also equivalent by (32) to $d X_{1}>0$. Therefore, " $d \mu_{0}>0$ or (non exclusive) $d X_{1}>0 "$ is equivalent to " $d \mu_{0}>0$ (and $d X_{1}>0$ )".

The following lemma will conclude the proof of Proposition 4.2.

Lemma 14 As a result of the date-T price rise, $d \mu_{t}>0$ and $d X_{t+1}>0$, for all $t=$ $0, \ldots, T-1$.

By Lemma 13, $d \mu_{0}>0$, and therefore, $d X_{1}>0$. Assume that for some $t=0, \ldots, T-2$, $d \mu_{t}>0$ and $d X_{t+1}>0$; we will show that this implies $d \mu_{t+1}>0$ and $d X_{t+2}>0$.

First consider the situation where $x_{t+1}=0$ before and after the change in price. In that case, by (58), $\mu_{t}=\mu_{t+1}$, which immediately shows that $d \mu_{t+1}>0$. Also, the differentiation of (32) with $d x_{t+1}=0$ implies $d X_{t+2}=d X_{t+1}>0$.

Second, consider that $x_{t+1}$ was strictly positive and remains so after the change. In that case, (55) holds at $t+1$. Suppose, as an assumption to be contradicted, that $d \mu_{t+1} \leq 0$. On the one hand, by the total differentiation of (55) at date $t+1, d \mu_{t+1} \leq 0$ is equivalent to

$$
d x_{t+1} \geq \frac{-\frac{\partial^{2} C_{t+1}}{\partial x \partial X}}{\frac{\partial^{2} C_{t+1}}{\partial x^{2}}} d X_{t+1}
$$

On the other hand, totally differentiating (60) at $t+1$ and substituting for $d \mu_{t+1}$ from (55) yield

$$
d \mu_{t}=-\left[\frac{\partial^{2} C_{t+1}}{\partial x^{2}}+\frac{\partial^{2} C_{t+1}}{\partial x \partial X}\right] d x_{t+1}-\left[\frac{\partial^{2} C_{t+1}}{\partial X \partial x}+\frac{\partial^{2} C_{t+1}}{\partial X^{2}}\right] d X_{t+1}
$$

where the first term between brackets on the right-hand side is strictly positive by assumption (30). Using (82) and simplifying it follows that

$$
d \mu_{t} \leq\left(\frac{\partial^{2} C_{t+1}}{\partial x^{2}}\right)^{-1}\left[\left(\frac{\partial^{2} C_{t+1}}{\partial x \partial X}\right)^{2}-\frac{\partial^{2} C_{t+1}}{\partial x^{2}} \frac{\partial^{2} C_{t+1}}{\partial X^{2}}\right] d X_{t+1}
$$


where assumption (48) implies that the coefficient of $d X_{t+1}$ is strictly negative. Thus the lemma's assumption that $d X_{t+1}>0$ implies $d \mu_{t}<0$, which contradicts the other lemma's assumption that $d \mu_{t}>0$. Thus the maintained assumption $d \mu_{t+1} \leq 0$ implies a contradiction and one must conclude that $d \mu_{t+1}>0$.

We still have to show that $d X_{t+2}>0$. By (55), the rise $d \mu_{t+1}>0$ requires a strict reduction in $\frac{\partial C_{t+1}}{\partial x}$, that is

$$
d x_{t+1}<\frac{-\frac{\partial^{2} C_{t+1}}{\partial x \partial X}}{\frac{\partial^{2} C_{t+1}}{\partial x^{2}}} d X_{t+1} .
$$

By assumptions (30) and (48), the coefficient of $d X_{t+1}$ in the above inequality is strictly positive and lower than unity. Since $d X_{t+1}>0$ as maintained assumption, it follows from (85) that $d x_{t+1}<d X_{t+1}$. The latter inequality finally implies by (32) that $d X_{t+2}>0$.

Given that the maintained assumption $d \mu_{t}>0$ and $d X_{t+1}>0$ is satisfied at $t=0$ (Lemma 13), Lemma 14 has been proven both when extraction $x_{t+1}$ is and remains null or when it is and remains strictly positive. By continuity (see the application of the Maximum Theorem in Footnote 39), it follows that Lemma 14 also holds across cases, that is when $x_{t+1}$ becomes null or becomes strictly positive following the price rise.

Let us now sum up the results. As a consequence of the rise in $p_{T}, X_{t}$ increases by Lemma 14, implying that cumulative supply $X^{0}-X_{t}$ decreases, at all dates $t=1, \ldots, T$. By the law of supply (Lemma 11), date- $T$ instantaneous supply $x_{T}$ not only increases but increases in such a way that the subsequent cumulative supply $X^{0}-X_{T+1}$ is higher than before the price rise. However instantaneous supply $x_{t}$ decreases at all subsequent dates $t>T$ by Lemma 10. Hence, defining cumulative supply as excluding date- $T$ supply yields the second point of Proposition 4.

\section{B Multiple Deposits with Endogenous Production Periods}

As mentioned in Subsection 4.b, when development dates $\tau^{j}, j=1, \ldots, J$, are fixed, the problem for each individual deposit $j$ is identical to the single-deposit problem of Section 2. By Proposition 1, for each independent deposit $j=1, \ldots, J$, and thus at the aggregate level, an exogenous rise in exploitable reserves increases restricted supply at all dates $t \geq \tau^{j}$, and a price rise at some date $T>0$ reduces restricted (short run) and unrestricted (medium run) supply of active deposits at all dates $\tau^{j} \leq t<T$.

Therefore in the sequel, we analyze the long run, when both exploitable reserves $X^{j}$ and development dates $\tau^{j}$ are choice variables, $j=1, \ldots, J$. Since the exploitation of each deposit is independent of other deposits, let us focus on deposit $j$. Consider at this stage the development date $\tau^{j}$ as given. Conditional on $\tau^{j}$, optimum extraction flows $x_{t}^{j}\left(p, \tau^{j}\right)$ for all $t \geq \tau^{j}$ are determined by (44) where $\lambda^{j}=\lambda^{j}\left(p, \tau^{j}\right)$ will be characterized shortly.

On the one hand, $\lambda^{j}\left(p, \tau^{j}\right)$ reflects the contribution of marginal reserves at the producer's optimum. Indeed, denoting deposit- $j$ 's value function conditional on $\tau^{j}$ by

$$
\begin{aligned}
& \mathcal{V}^{j}\left(p, \tau^{j}, X^{j}\right) \equiv \max _{\left(x_{t}^{j}\right)_{t \geq \tau^{j}}} \sum_{t \geq \tau^{j}} p_{t} x_{t}^{j}-C_{t}^{j}\left(x_{t}^{j}\right) \\
& \text { subject to } \sum_{t \geq \tau^{j}} \quad x_{t}^{j} \leq X^{j}
\end{aligned}
$$


and keeping in mind that $\lambda^{j}\left(p, \tau^{j}\right)$ is formally the Lagrange multiplier associated with constraint (87), the Envelope Theorem for constrained problems implies

$$
\lambda^{j}\left(p, \tau^{j}\right)=\frac{\partial \mathcal{V}^{j}\left(p, \tau^{j}, X^{j}\left(p, \tau^{j}\right)\right)}{\partial X^{j}},
$$

which relates the optimum amount of reserves $X^{j}\left(p, \tau^{j}\right)$ with their implicit value $\lambda^{j}\left(p, \tau^{j}\right)$. In equation (88), $\frac{\partial \mathcal{V}^{j}\left(p, \tau^{j}, X^{j}\right)}{\partial X^{j}}$ is a decreasing function of reserves $X^{j}$ by the assumption that extraction costs are strictly convex. Moreover, it is a decreasing function of $\tau^{j}$, since exploiting unchanged reserves $X^{j}$ over a smaller set of dates means that marginal reserves must optimally be extracted at higher costs.

On the other hand, (45) indicates that exploitable reserves $X^{j}\left(p, \tau^{j}\right)$ are optimally produced in such a way as to equate their marginal development cost $E_{\tau^{j}}^{j \prime}\left(X^{j}\left(p, \tau^{j}\right)\right)$ with their implicit valuation underground $\lambda^{j}\left(p, \tau^{j}\right)$. This reserve-supply relation is strictly increasing since the $E_{\tau^{j}}^{j}$ function is strictly convex by assumption.

Thus in optimum, for a given development date $\tau^{j}$, the rent $\lambda^{j}\left(p, \tau^{j}\right)$ and reserves $X^{j}\left(p, \tau^{j}\right)$ are jointly determined by the combination of (88) with (45):

$$
\frac{\partial \mathcal{V}^{j}\left(p, \tau^{j}, X^{j}\left(p, \tau^{j}\right)\right)}{\partial X^{j}}=\lambda^{j}\left(p, \tau^{j}\right)=E_{\tau^{j}}^{j \prime}\left(X^{j}\left(p, \tau^{j}\right)\right)
$$

\section{2.a Proof of Lemma 1}

The optimum rent $\lambda^{j}\left(p, \tau^{j}\right)$ and optimum reserves depend on the (at this stage) exogenous development date $\tau^{j}$. As explained above, $\frac{\partial \mathcal{V}^{j}\left(p, \tau^{j}, X^{j}\right)}{\partial X^{j}}$ is decreasing in $\tau^{j}$. Let $\tau^{j \prime}<\tau^{j}$ be two exogenous development dates: it must be that $\frac{\partial \mathcal{V}^{j}\left(p, \tau^{j}, X^{j}\right)}{\partial X^{j}} \geq \frac{\partial \mathcal{V}^{j}\left(p, \tau^{j}, X^{j}\right)}{\partial X^{j}}$. Moreover, by assumption (41), $E_{\tau^{j}}^{j \prime}\left(X^{j}\right) \geq E_{\tau^{j}}^{j \prime}\left(X^{j}\right)$. It thus follows that (89) taken at $\tau^{j}$ and at $\tau^{j \prime}<\tau^{j}$ generates two different rents such that

$$
\lambda^{j}\left(p, \tau^{j \prime}\right) \geq \lambda^{j}\left(p, \tau^{j}\right), \tau^{j \prime}<\tau^{j} .
$$

For any given development date $\tau^{j}$, the Maximum Theorem applies. ${ }^{40}$ Thus price changes continuously affect all variables and functions. In particular, if $\tau^{j}$ is the optimum development date, small changes in price that do not require any change in that date have continuous effects.

Consider now that $\tau^{j}=\arg \max _{\tau \geq 0} \mathcal{V}^{j}\left(p, X^{j}(p, \tau), \tau\right)-E_{\tau}^{j}\left(X^{j}(p, \tau)\right)$, where, given $\tau$, $X^{j}(p, \tau)$ is chosen optimally as described above. It follows that, for any $\tau^{j \prime} \neq \tau^{j}$,

$$
\mathcal{V}^{j}\left(p, X^{j}\left(p, \tau^{j}\right), \tau^{j}\right)-E_{\tau^{j}}^{j}\left(X^{j}\left(p, \tau^{j}\right)\right)-\left[\mathcal{V}^{j}\left(p, X^{j}\left(p, \tau^{j \prime}\right), \tau^{j^{\prime}}\right)-E_{\tau^{j \prime}}^{j}\left(X^{j}\left(p, \tau^{j \prime}\right)\right)\right] \geq 0 .
$$

\footnotetext{
${ }^{40}$ When reserves are endogenously determined at the exogenous date $\tau^{j}$, the optimal reserves $X^{j}\left(p, \tau^{j}\right)$ must be finite so that the extraction possibility set is bounded, and is evidently closed. Prices in $p$ also affect the objective (42) continuously. Furthermore, this objective is strictly concave by assumption and the set of extraction possibilities is convex since the convex combination of two possible extraction paths satisfying the exhaustibility constraint (40) satisfies the same constraint. The Maximum Theorem thus applies: given the development date $\tau^{j}$, the optimum sequence of extraction $x_{t}^{j}\left(p, \tau^{j}\right)$ and the multiplier $\lambda^{j}\left(p, \tau^{j}\right)$ evaluated at the optimum are continuous functions of each price in the vector $p$.
} 
The first part of the lemma considers a price rise at some date $T$, posterior to the deposit-opening date $\tau^{j}$. Assuming instead that $T \leq \tau^{j \prime}<\tau^{j}$, let us show now that no increase in price can cause (91) to be violated, so that a price rise cannot cause the development date to be accelerated. Precisely, consider an infinitesimal change in price at date $T>\tau^{j}$. Denoting by $\Delta$ the total derivative of the left-hand side of inequality (91), one obtains

$$
\begin{aligned}
\Delta & =\frac{\partial \mathcal{V}^{j}\left(p, X^{j}\left(p, \tau^{j}\right), \tau^{j}\right)}{\partial p_{T}}+\frac{\partial \mathcal{V}^{j}\left(p, X^{j}\left(p, \tau^{j}\right), \tau^{j}\right)}{\partial X^{j}} \frac{d X^{j}\left(p, \tau^{j}\right)}{d p_{T}}-E_{\tau^{j}}^{j \prime}\left(X^{j}\left(p, \tau^{j}\right)\right) \frac{d X^{j}\left(p, \tau^{j}\right)}{d p_{T}} \\
& -\left[\frac{\partial \mathcal{V}^{j}\left(p, X^{j}\left(p, \tau^{j \prime}\right), \tau^{j \prime}\right)}{\partial p_{T}}+\frac{\partial \mathcal{V}^{j}\left(p, X^{j}\left(p, \tau^{j \prime}\right), \tau^{j \prime}\right)}{\partial X^{j}} \frac{d X^{j}\left(p, \tau^{j \prime}\right)}{d p_{T}}-E_{\tau^{j \prime}}^{j \prime}\left(X^{j}\left(p, \tau^{j \prime}\right)\right) \frac{d X^{j}\left(p, \tau^{j \prime}\right)}{d p_{T}}\right] .
\end{aligned}
$$

Recalling the second equality in (89), we have

$$
\frac{\partial \mathcal{V}^{j}\left(p, X^{j}\left(p, \tau^{j}\right), \tau^{j}\right)}{\partial X^{j}} \frac{d X^{j}\left(p, \tau^{j}\right)}{d p_{T}}-E_{\tau^{j}}^{j \prime}\left(X^{j}\left(p, \tau^{j}\right)\right) \frac{d X^{j}\left(p, \tau^{j}\right)}{d p_{T}}=0
$$

and similarly for $\tau^{j \prime}$. Thus, $\Delta$ may be rewritten

$$
\Delta=\frac{\partial \mathcal{V}^{j}\left(p, X^{j}\left(p, \tau^{j}\right), \tau^{j}\right)}{\partial p_{T}}-\left[\frac{\partial \mathcal{V}^{j}\left(p, X^{j}\left(p, \tau^{j \prime}\right), \tau^{j \prime}\right)}{\partial p_{T}}\right],
$$

where the Envelope Theorem applied to (86)-(87) implies

$$
\frac{\partial \mathcal{V}^{j}\left(p, X^{j}\left(p, \tau^{j}\right), \tau^{j}\right)}{\partial p_{T}}=x_{T}^{j}\left(p, \tau^{j}\right)
$$

for $\tau^{j}$ and for $\tau^{j \prime} . \Delta$ thus reduces to

$$
\Delta=x_{T}^{j}\left(p, \tau^{j}\right)-x_{T}^{j}\left(p, \tau^{j \prime}\right)
$$

Finally, the inequality (90) established above, together with the first-order condition (44) characterizing extraction at date $T$ implies that $x_{T}^{j}\left(p, \tau^{j}\right) \geq x_{T}^{j}\left(p, \tau^{j \prime}\right)$, which proves that

$$
\Delta \geq 0
$$

Since a price rise positively affects the left-hand side of inequality (91), it cannot cause the earlier date $\tau^{j \prime}<\tau^{j}$ to become the optimal development date.

The second part of the lemma considers a price rise occurring at date $T$ prior to the deposit-opening date $\tau^{j}$. Under the initial prices, assume that extraction takes place at dates $t \geq \tau^{j} \geq T$ and reserves are optimally developed at $\tau^{j}$; that must yield higher intertemporal profits (42) than opening the deposit at a later date. A postponing of the opening date $\tau^{j}$ to $\tau^{j^{\prime}}>\tau^{j}$ would restrict extraction to dates $t \geq \tau^{j \prime}>\tau^{j}$, over which price conditions are unchanged by the rise in price at $T$. Clearly, that restriction cannot dominate the possibility of extracting at the larger set of dates $t \geq \tau^{j}$. 


\section{2.b Proof of Proposition 5}

The first two points of the proposition are shown in the main text, as well as the first part of the third point; there remains the second part of the third point. We need to show that, if the development-cost function $E_{\tau^{j}}^{j}$ is time autonomous, then a price rise at $T>0$ reduces long-run aggregate supply at all dates $t \neq T$ as in the second point of the proposition.

Assume that the deposit-development date $\tau^{j}$ is endogenous. When the function $E_{\tau^{j}}^{j}$ does not depend on $\tau^{j}$, the Maximum Theorem applies as in Footnote 40 even though $\tau^{j}$ is endogenous; the objective (42) is continuous in price parameters because a price change, even if it causes a change in $\tau_{j}$, does not affect the development technology encompassed in the $E^{j}$ function.

By the Maximum Theorem, a rise in price continuously affects extraction $x_{t}^{j}(p)$; it follows that the results established for a fixed opening date carry over when the opening date may change as a result of a price rise. 